Contraindre, ou le rôle funèbre du jādu pațuā, « peintre-magicien » (Bengale, Inde)

The jādu pațuā's Coercive Role (Bengal, India)

\title{
Rosita De Selva
}

\section{OpenEdition}

1 Journals

Édition électronique

URL : http://journals.openedition.org/span/1360

DOI : $10.4000 /$ span. 1360

ISSN : 2268-1558

Éditeur

École pratique des hautes études. Sciences humaines

\section{Édition imprimée}

Date de publication : 1 décembre 1994

Pagination : 43-87.

ISSN : 0294-7080

\section{Référence électronique}

Rosita De Selva, "Contraindre, ou le rôle funèbre du jādu pațuā, " peintre-magicien » (Bengale, Inde) », Systèmes de pensée en Afrique noire [En ligne], 13 | 1994, mis en ligne le 05 mars 2014, consulté le 20 avril 2019. URL : http://journals.openedition.org/span/1360 ; DOI : 10.4000/span.1360 


\section{CONTRAINDRE, OU LE RÔLE FUNÈBRE DU JĀDU PATUA ' ${ }^{1}$, PEINTRE-MAGICIEN" \\ (Bengale, Inde)}

\section{par}

\section{Rosita de Selva}

Dès qu'il apprend qu'un décès a eu lieu, le jādu patuā se dirige vers la maison du mort pour y tenir son rôle. Deux hommes sont concernés par cette visite: le père, défunt, et le fils aîné, survivant. L'art du patuā consiste à recréer la contrainte de deuil qui lie ces deux personnages. La pratique du jădu patuã a en effet ceci de particulier qu'elle ne fait pas partie des rites que les parents du défunt doivent effectuer après la mort de l'un des leurs. Les parents font même tout pour éviter la venue du personnage. S'il ne se présente pas, le cycle funéraire s'accomplit tout à fait normalement, l'ordre sacrificiel est

1 Mot-à-mot: "magie-peintre". Pour la transcription du bengali, nous avons suivi, en les simplifiant, les règles adoptées par S. K. Chatterjee (1975). Les noms de lieu sont transcrits selon leur orthographe usuelle au Bengale. Le travail présenté ci-dessous est né de la confrontation de faits relatifs à l'Inde avec les recherches menées sur le deuil et le fétiche par le laboratoire Systèmes de Pensée en Afrique noire. Nous remercions $\mathrm{D}$. Liberski, qui nous a incité à rédiger ces lignes, ainsi que l'équipe "Rites et pratiques socio-religieuses" du Centre d'Etudes Indiennes, où nous avons exposé nos matériaux.

Le deuil et ses rites III

Systèmes de pensée en Afrique noire, 13, 1994 
rétabli ${ }^{2}$, les femmes peuvent à nouveau porter le vermillon, la bière de mil peut à nouveau être consommée. La contrainte créé par le patuă s'exerce donc en marge du système rituel qui règle le deuil ${ }^{3}$.

$J \bar{a} d u$ est un nom commun (ici en apposition); il est géneralement traduit par "magie". Patuã, habituellement traduit par "peintre", est forme de la racine pat ("tissu", "peinture sur tissu", "image") et du suffixe $u \bar{a}$, qui indique une relation ${ }^{4}$. Le patuā est donc, littéralement, "[celui] qui a rapport au pat" 5 .

En quoi le jădu patuã qui se présente devant la maison en deuil a-t-il "rapport au pat" et qu'est-ce qui lui vaut le nom de magicien ? Pour sa visite funebre, le jädu patuã emporte, serrées en un rouleau qui ne dépasse pas la grandeur d'une main, une série de petites peintures sur papier, représentant chacune, de manière extrêmement sommaire, une figure humaine et quelques objets, parfois des animaux et un être poilu à l'aspect quelque peu repoussant. Le visage humain, dessiné de profil, présente une caractéristique remarquable: l'œil, de grande dimension, ne comporte pas de prunelle. Il est, en quelque sorte, vide de regard. La première mention un tant soit peu détaillée

2 A mesure que le deuil est levé, le village d'abord, les parents du défunt ensuite, reprennent l'exécution des sacrifices, suspendus pendant le temps du deuil (Archer, 1974: 335-337). La société dont il est question, nous le verrons, n'est pas hindoue.

${ }^{3}$ L'emploi à l'égard du patuã de l'adjectif funèbre (plutôt que funéraire) tente d'exprimer ce trait. "Funèbre" a en effet successivement pour sens: 1) "ce qui a rapport à la mort" 2) "ce qui a trait aux funérailles". "Funéraire" a bien aujourd hui les mêmes sens, mais l'ordre en est inversé (Trésor de la Langue Française 19811990; Grand Robert 1986). "Funéraire" fait donc d'abord penser à l'ensemble organisé de rites qu'une société donnée a élaborés pour traiter la mort. Or, la pratique du patuă ne rentre pas dans ce cadre. Nous verrons par ailleurs que le dernier sens de funèbre, à savoir "ce qui évoque l'idée de la mort", convient bien au jädu patuã.

${ }^{4}$ Cf. S.K. Chatterjee, 1975: 679. Jädu se dit aussi yädov, ou jädo.

${ }^{5}$ Le patuā est aussi connu sous le nom de citrakar, "celui qui fait le citra" (citra= "image"). Nous nous limitons volontairement ici à l'appellation patuā. En effet, seul ce terme (ou ses variations sur la même racine: poto, patkār, patidar, etc.) est utilisé dans le contexte qui nous intéresse ici, à savoir celui de la présentation de pat. Ajoutons que si citrakar apparait dans de nombreux cas comme un "doublet" de patuá (notamment comme nom propre ou comme désignation de caste), des deux termes: citrakar et patuã, seul le second peut être associé à jädu (on ne parle jamais de jãdu citrakar). 


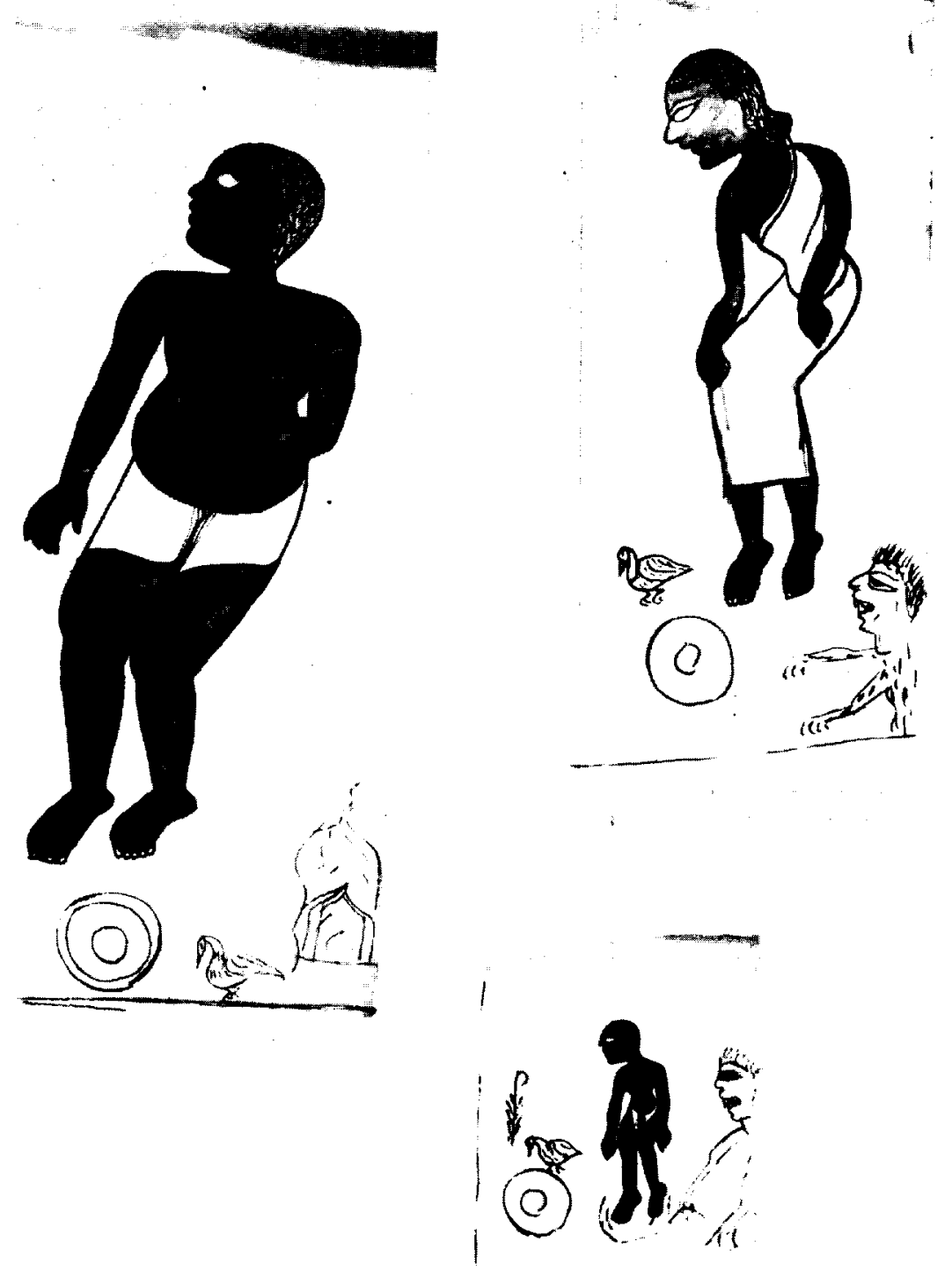

pat mortuaires d'un homme, d'une femme, d'un enfant 
de l'utilisation de ces pat est donnée en 1932 par G. Dutt, un haut fonctionnaire bengali de l'administration (anglaise) de l'époque qui, comme beaucoup de ses contemporains des milieux intellectuels, s'intéressait à la "culture populaire" de son pays. Il écrit:

"Le Jādu Patuā se présente devant la maison de la famille en deuil avec une esquisse du défunt faite d'imagination. Il n'y a pas d'effort de ressemblance; l'image consiste en un dessin (angl. drawing) très simple, d'adulte ou d'enfant, d'homme ou de femme, selon l'âge et le sexe du défunt. Le Jādu Patuā présente cette image complètement dessinée, à l'exception d'un élément: l'iris de l'œil. Il montre l'image aux parents et leur dit que le défunt est en train d'errer en aveugle dans l'autre monde, et qu'il continuera à le faire jusqu'à ce qu'ils envoient des présents d'argent ou quelque autre chose à travers lui, le Jādu Patuā, de façon à ce qu'il (souligné par nous) puisse effectuer l'acte du Chakshudana ou don de la vue (bestowal of eyesight)... [Les parents du défunt] croient que c'est vrai... Ils protestent qu'ils lui ont déjà fait des dons au moment de sa mort mais le Jādu Patuā reste inflexible; il leur dit que le Roi Yama [le dieu des morts] a pris ces dons... et qu'ils doivent donc lui envoyer de nouvelles choses à travers lui. Les parents font alors au Jādu Patuā des présents d'argent ou d'autres objets à usage domestique pour qu'il les transmette au mort, et le Jādu Patuā termine l'image en effectuant l'acte du Chakshudana, ou report de l'iris sur l'image du défunt. C'est peut-être de cette pratique semimagique que le Jādu Patuā tient son nom (Jādu - magie; Patuā peintre)". (G. Dutt, 1932, traduit de l'anglais par nous).

La littérature sur les jādu patuã est aujourd'hui relativement abondante, mais le texte de G. Dutt reste l'un des plus complets, aussi bien sur la pratique effective du jādu patuã que sur la façon dont elle est perçue par les Bengalis. Quarante-cinq ans plus tard, Mildred Archer ${ }^{6} s^{\prime}$ exprime dans les mêmes termes, en ajoutant:

"à côté des figures humaines étaient dessinés en petit divers objets, tels qu'une vache, une chèvre, des poulets, des plats en cuivre, des ornements ou de l'argent [...] Prenant ces images (angl. pictures),

${ }^{6} \mathrm{M}$. Archer est connue pour ses travaux sur la peinture populaire de l'Inde, particulièrement de l'est de l'Inde; le texte qui suit est un extrait d'un livre où elle présente différents types de peintures recueillies par son mari, W. G. Archer, haut fonctionnaire en Inde de 1940 à 1946. 
le jadupatua se rendait au village où était survenu le décès... Il demandait qui était mort, effectuait un rapide calcul de l'état des ressources familiales et présentait l'image adéquate. Il expliquait aux parents que le défunt était comme un aveugle en train d'errer dans l'autre monde mais que la vue pourrait lui être redonnée si lui, le jadupatua, redonnait à l'oil vide sa pupille. Il serait prêt à leur rendre ce service en échange (souligné par nous) des objets indiqués sur le dessin... Ayant reçu son paiement, ... [il] s'en allait en emportant son butin" (angl. "spoils") ${ }^{7}$ (traduit de l'anglais par nous, Archer, 1977: 16).

Nous avons effectué au Bengale plusieurs enquêtes sur la pratique funèbre du jadupatua $\tilde{a}^{8}$. Comme beaucoup, nous nous interrogions sur ce qui pousse les proches du mort à s'exécuter, le but du patuā n'étant pas, semble-t-il, tout-à-fait celui que les intéressés prêtent à son action. A la suite de G. Dutt, les auteurs disent simplement: la famille du défunt "croit que c'est vrai". Plusieurs évoquent le sentiment de crainte qui agite les parents: "les craintes... au sujet des morts sont si fortes que la demande [du patuā] ne sera jamais refusée" (Faivre, 1980: 120) ${ }^{9}$. Son nom de "magicien" viendrait de là dit M. Archer: "les jadupatua sont redoutés; pour cette raison on en est venu à désigner leur caste sous le nom de [...] peintres-magiciens" (1977: 16). Certains auteurs le considèrent comme un "prêtre". On établit aussi entre le jādu patuā et le brahmane - officiant rituel par excellence dans l'hindouisme - une relation plus ou moins explicite, et les jādu patua déclarent souvent: "nous sommes les brahmanes [de nos "clients"]". Enfin, l'idée que le jădu patuā n'est qu'un charlatan, dont la duplicité

7 "Spoils", généralement traduit par "butin" (cf. notamment Faivre, 1980: 120) a également le sens de "dépouilles", ce qui, dans le contexte présent, mérite assurément réflexion. Le sens littéral est "ce qui a été gâté", ou "souillé".

${ }^{8}$ Lors d'un long séjour au Bengale (1973-1977) pour partie pris en charge par le Ministère des Affaires Etrangères (1 an) et l'ORSTOM (1 an). Je remercie ces institutions, la première pour $m$ 'avoir donné le goût de l'Inde, plus particulièrement du Bengale et de sa langue, la seconde pour m'avoir permis de poursuivre plus efficacement mes recherches sur les patuã.

9 J. B. Faivre était un artiste. Au cours d'un voyage en Inde, très frappé par les peintures des jâdu patuã et la dimension religieuse de cet "art", il décida de vivre parmi eux, ce que peu de gens avant lui avaient fait. 
n'a d'égale que la simplicité de ceux à qui il s'adresse, doit être signalée. "La clientèle du patuā est rurale et illettrée"... "Ce sont surtout les femmes qui y croient"... "Eux y croient" (sous entendu: nous, nous n'y croyons pas et nous ne nous laisserions pas prendre à de tels stratagèmes).

Il semble évident que le patuā cherche à jouer de la crainte qu'inspire un mort qui n'aurait pas été traité comme il faut. Une rapide enquête de terrain montre toutefois que les parents du mort sont, en règle générale, loin d'être aussi crédules qu'on le laisse entendre. Bien entendu, ils savent que les objets emportés par le patuă ne reviennent qu’à lui seul. Qu'est-ce qui fait, alors, qu'ils se plient à ses exigences ? Les auteurs accordent une grande importance (et on peut les comprendre) au geste du "don de l'(xil", ce qui place l'image au centre du dispositif déployé par le patuã. "Cet ail peint ne peut manquer d'impressionner, si l'on pense à l'ancienneté du geste et de sa valeur magique" dit J. B. Faivre (1980: 120). Pour M. Archer, "C'est cet acte du don de l'œil qui vaut au jadupatua son nom de magicien" (1977: 16). On peut toutefois se demander comment le fait de représenter un visage sans pupille peut suffire à assurer les parents que le défunt erre dans l'au-delà ? Dans un autre registre, on peut également se demander en quoi les présents offerts par la famille permettraient au patuā d'agir sur la faculté de "voir" du défunt (nous verrons à ce sujet que ce n'est pas parce qu'il peint la pupille que le patuã reçoit des objets, mais parce qu'il recueille des objets qu'il peut peindre la pupille, logique qui avait déjà été saisie par G. Dutt). Y a-t-il par ailleurs un lien entre le fait de voir le pat (pour les survivants) et l'idée que la vue est rendue au défunt? Pourquoi cette capacité à voir (rendue au défunt, ou donnée à la famille du mort ?) fait-il cesser une fois pour toutes les réclamations de Yama?

Dans le présent travail, nous verrons que la présentation de l'image et le geste désigné comme "don de l'cil" sont précédés d'une série de comportements et de paroles précises. Si Dutt avait bien noté l'existence de ces paroles, elles n'ont cependant jamais été présentées dans la littérature, et ni les circonstances qui les entourent, ni le moment de leur énonciation n'ont jamais été pris en compte. L'examen de ces préliminaires - auquel nous limiterons le présent exposé - fait 
apparaître des traits qui conduisent à reconsidérer les raisons qui font du jădu patuã un "magicien", sans rien ôter toutefois à celles qui aboutissent à construire à son propos l'image d'un "peintre" d'une part, d'une sorte de brahmane d'autre part. La perspective sur le personnage s'en trouve singulièrement élargie. Voyons d'abord rapidement comment le jādu patuã est rapproché du brahmane et de quelle façon il est un "peintre".

\section{Le jādu patuā et le brahmane}

Le terme caksudăn - ou "don de l'œil" - qui, dans certains contextes, est utilisé pour désigner le geste du jädu patuã correspond au sanscrit nayanamilan, "ouverture de l'œil". Ce terme renvoie à un contexte rituel hindou très précis; il désigne le geste effectué par le brahmane sur l'image de la divinité lors du rite hindou dit "d'installation" (beng. pratistha karā) de cette divinité sur le lieu et pour le temps où elle sera appelé à exercer sa puissance. Le terme s'applique également à la séquence du rite où vient s'insérer ce geste. Ce rite est pratiqué dans toute l'Inde. Il y est attesté depuis longtemps. Il est décrit et commenté dans plusieurs traités sanscrits et bien connu des indianistes. Il est aujourd'hui encore régulièrement effectué. A la différence de la pratique du jādu patuã qui, nous le verrons, est difficile à voir, il est aisément observable; c'est un rite tout à fait public. Il peut être pratiqué sur une effigie destinée à demeurer de manière permanente en un lieu (un temple par ex.), ou confectionnée pour le temps d'un rite donné; au terme de la période rituelle, l'image sera alors immergée. Cette image peut être une sculpture (en matériaux divers; souvent façonnée en terre crue si elle est temporaire), mais aussi une peinture. Dans le cas d'une effigie nouvellement confectionnée, celle-ci arrive sur le lieu de culte richement peinte et décorée par les artisans concernés: sütradhar (charpentiers, litt. "ceux qui tiennent le fil"), citrakar (litt. "ceux qui font l'image"), kumbhakar ("faiseurs de pots"), malakār ("faiseurs de guirlandes"), etc... Toutefois, de ses yeux, à la réalisation desquels a été apporté un soin tout particulier, on dit qu'ils ne voient pas. Cette faculté sera "activée" par le brahmane 
lors du rite du caksudān, lors des rites préliminaires que ce dernier effectue devant l'effigie et destinés à rendre la divinité - dite encore "en sommeil" - présente sur les lieux du culte. C'est seulement après que la divinité a été ainsi "éveillée" par le brahmane que pourra commencer l'exécution de la célébration proprement dite ${ }^{10}$.

L'univers où s'exerce l'art du jādu patuā est tout autre. On peut dire, en reprenant une terminologie courante, qu'il est "non brahmanique". Le jădu patuã s'adresse, nous le verrons, à des non Hindous (ce qui ne veut pas dire que la logique brahmanique ne soit pas a l'auvre dans sa pratique, nous y reviendrons). Lors de la présentation de pat, et de manière générale lorsqu'un discours sur le patuā est émis dans le contexte qui est le sien sans que viennent s'y mêler de connotations brahmaniques, le terme caksudān n'est guère utilisé. Le fait de placer la pupille dans l'ceil de la figure ne fait l'objet d'aucune appellation précise, et il n'est pas, de manière générale, mis en avant. Si toutefois l'on y fait référence, on dit alors que le patuă fait là du jādo, terme qui désigne à la fois l'ensemble de la pratique et le personnage.

Malgré tout, il n'est pas douteux que pour les Bengalis, voire pour bon nombre d'Indiens, le geste du patuã évoque immédiatement le geste du brahmane. Que l'on établisse un rapport entre les deux scènes ressort de maints discours. L'emploi du terme caksudán pour désigner l'acte du patuā n'en est qu'une des marques.

Le jādu patuā est également rapproché du brahmane parce qu'il traite de la mort. Avant de développer ce point, il faut présenter le contexte où se fait la présentation de pat. Le jâdu patuã exerce en une aire géographique bien déterminée: dans une partie de l'État indien du Bengale (sur la frange ouest des districts de Purulia, Bankura, Bardhamān, Birbhum et Medinipur) et au Bihar (notamment dans le district des Santals Parganas) ${ }^{11}$. Cette zone forme une large bande

${ }^{10}$ Pour la description de ces rites préliminaires, cf. notamment Renou, 1947, I: 572-73. Pour le rite effectué sur des peintures, voir Talwar, 1979: 106-107 (Orissa), et Ostör, 1980 (Bengale).

11 L'Inde est une fédération d'états; chacun est subdivisé en "districts". Le jâdu patuā s'aventure rarement, ou seulement pour de courtes périodes, en dehors de l'aire géographique mentionnée ci-dessus. 
entourant à l'ouest et au nord le delta du Gange. Le relief y est relativement accidenté, la forêt gagne sur les cultures, les étangs et bassins se font plus rares, la population est moins dense que dans la plaine voisine. Seule l'étroite vallée de la rivière Damodar qui, venant du nord-ouest, rejoint le Gange, vient interrompre cette sorte de barrière de collines "sèches" et "boisées" qui, aux yeux d'un Bengali, sépare le "Bengale d'or" (sonār bãnglā), delta riche et peuplé qu'il considère comme sien, du reste de l'Inde. C'est donc en une zone frontière aux yeux des Bengalis qu'évoluent les jādu patuã. Cette région est depuis plusieurs siècles la terre d'élection des Santals, l'un des principaux groupes "tribaux" de l'est de l'Inde ${ }^{12}$. Les Santals forment un groupe endogame. Ils ne sont ni hindous, ni musulmans. Ils vivent en grande partie en zone rurale, dans des villages qui leur sont propres. Ils ont gardé une forte identité, tout en ayant des contacts anciens et réguliers avec les groupes voisins (aujourd'hui essentiellement hindous ou musulmans, mais anciennement également jaïns et bouddhistes). La société santale est fondée sur un ensemble de mythes et de rites propres, même si l'on y retrouve des éléments communs aux autres systèmes socio-religieux de l'Inde ${ }^{13}$. L'influence brahmanique y est moins sensible que partout ailleurs. Cette société n'est notamment pas organisée selon le système de castes. Les rites y sont conduits par des officiants autres que le brahmane (ce sont en général des Santals, mais pas exclusivement). Notons aussi que, bien qu'ils occupent des terres qui, géographiquement et administrativement parlant, sont rattachées depuis plusieurs siècles au Bengale, les Santals ne sont pas considérés comme Bengalis. Ces derniers, qu'ils soient hindous ou musulmans, les situent comme étant extérieurs à leur société et les considèrent comme impurs.

12 Les "tribus" (terme mis en usage par les Anglais), ou Adivãsi dans les langues de l'Inde (litt. "premiers habitants"), se distinguent du reste de la population indienne par un ensemble de caractères particuliers, notamment linguistiques, sociaux et religieux. Selon les recensements, qui continuent d'utiliser ce terme, ils constituent environ $9 \%$ de la population indienne.

13 Yama est par exemple également très présent dans l'eschatologie santale. 
C'est chez ces Santals que le jādu patuã se rend avec ses images funebres. Est-il lui-même santal, comme le pensent certains, et comme peut en effet le laisser croire le nom de sāntāli patuā qui, nous le verrons, lui est souvent donné ? Des observations simples montrent que non. Les quartiers patuā, toujours de petite dimension (six a dix maisons au plus) forment des ensembles bien distincts des villages santals (ils peuvent être totalement isolés ou situés aux abords des communautés hindoues ou musulmanes de statut similaire au leur). Les habitations proprement dites se différencient par ailleurs en bien des points de celles des Santals. Le plus souvent très délaissées, elles "souffrent mal la comparaison avec les très belles demeures santales, admirablement tenues" note J. B. Faivre (1980: 112). Enfin, la langue "maternelle" du patuā est le bengali. Les termes qui le désignent sont tous bengalis. Lui-même se reconnaît comme Bengali, et même si les avis different sur son statut ${ }^{14}$, on peut dire que le jădu patuă est bien, aujourd'hui, "caste" (qu'il soit, ou se veuille hindou ou musulman vient ici en second plan ${ }^{15}$ ).

Pour notre propos, nous retiendrons que le patuā n'est pas santal et qu'au sein de la société hindoue des castes, il a un statut fluctuant, plutôt bas, allant parfois jusqu'à la limite de l'exclusion (il est alors au fond très proche des Santals, sans être toutefois, comme ces derniers, totalement exclu du système).

J. B. Faivre écrit: "Basudev Citrakar dit que 'le [patuã] est le brahmane des Santals' car il officie lors du déces [...] Les objets dessinés au bas de la figure représentant le mort précisent les exigences du jădu patuā $[\ldots]$ butin que plusieurs [patuā] ont comparé au salaire, dān, du brahmane lors des cérémonies funéraires" (Faivre, 1981: 112 \& 120). C'est bien ce même message que l'on trouve dans le texte de

${ }^{14}$ On sait les fluctuations que peut connaître le statut d'une caste donnée. Nous ne pouvons ici entrer dans les détails. Indiquons seulement que les patuā sont tantôt (sous le nom de citrakar) assimilés aux "artisans" (silpi), lesquels sont, au Bengale, pleinement intégrés à la société des castes et ont un statut relativement honorable, et tantôt relégués à l'extérieur de cette société parmi les hors-castes ou "sans castes" (jäthin). lis sont parfois identifiés aux Mal et aux Bediya, groupes semi-itinérants et très mal connus, au statut très inférieur.

15 Nous verrons plus loin que certains palnā sont hindous, d'autres musulmans. 
M. Archer. Elle qualifie en effet de "service" le fait de placer la pupille dans l'œil aveugle de l'image, et les objets que le patuā reçoit constituent, dit-elle, un "paiement" effectué "en échange" de ce "service"16. Bien qu'il ne soit pas cité, l'analogie avec le prêtre brahmane qui officie lors des rites funéraires hindous est clairement suggérée. Au terme des rites qu'il effectue pour la famille d'un défunt hindou, cet officiant emporte des objets domestiques ayant appartenu au défunt. Même si ce brahmane occupe au sein de sa caste une position inférieure parce qu'il traite les morts, il n'en reste pas moins vrai que les objets qu'il reçoit sont bien les mêmes que ceux qui sont cités dans les textes canoniques hindous parmi les dons (dān) accordés aux brahmanes en échange des services rituels qu'ils rendent. Ces dons sont classés en trois catégories définies et hiérarchisées. La liste de ces objets est la suivante: "1) nourriture... 2) ustensiles domestiques... 3) ombrelle, siege, vaisselle..., tout ce qui est vieux et usage" (Toffin, 1990: 137, et Kane 1974: II, 843). Ce sont également, à peu de chose pres, ceux qui sont représentés sur le pat, et réclamés par le patuã.

On peut certes se demander comment peut être établie une relation entre la pupille apposée par un brahmane sur l'image d'une divinité et celle que le patuã pose sur l'image du défunt, comment le "donner l'ail" à la divinité peut être comparé au "donner l'œil" à un défunt. Nous soulignerons ici un seul point: dans la pratique du patuā, la représentation et la mort forment un couple indissociable. Notre but n'est pas d'examiner le bien-fondé du rapprochement entre le brahmane et le jādu patuă, de nous demander à quel type de brahmane ce dernier est comparable (on sait que tous les brahmanes ne sont pas

16 "Dans certains cas, un autre type d'honoraires (angl. fee) était soutiré" écrit-elle un peu plus loin. "La tradition veut que les restes des os du défunt santal soient immergés dans la rivière sacrée Damodar qui, dans la mythologie santale, représente la mer... [Comme cela est bien souvent impossible à réaliser] les Santal paient parfois le jädu pafuā pour aller jusqu’à la Damodar faire une immersion symbolique..." (Archer, 1977: 17). Pense-t-elle à l’immersion de l'image ? On observe en tous cas que, dans le stock d'images mortuaires du patuã, certaines comportent la fameuse "pupille" tandis que sur d'autres, cette pupille est comme "grattée", comme si on avait voulu l'effacer (pour une éventuelle réutilisation?). On peut donc penser que le patuā ne laisse pas l'image du mort à la famille. 
à mettre sur le même plan) ou par quel mécanisme le jādu patuăa, au statut excessivement bas, peut être assimilé à celui qui a le statut le plus élevé dans la hiérarchie hindoue, ni de s'interroger sur le statut des objets donnés à l'un et à l'autre. Nous ne chercherons pas non plus à savoir à quel univers le jādu patuã emprunte ses outils ${ }^{17}$. On relèvera juste que, en s'assimilant ou en étant assimilé à un brahmane (quel qu'il soit), le jādu patuā est présenté comme un agent rituel faisant partie de l'ordre brahmanique ${ }^{18}$. Tout se passe dès lors comme s'il tenait son autorité de cet ordre.

Rappelons-nous cependant que les Santals sont gens de "tribus". Si le patuã et le brahmane sont bien d'un certain point de vue comparables et comparés (on se fonde sur le fait qu'ils sont tous deux effectivement présents à la mort et que l'un comme l'autre reçoivent des "honoraires" pour un "service" dont le bénéfice rituel leur est extérieur), leur position par rapport à ceux à qui ils s'adressent est donc bien différente. Dans un cas (celui du brahmane) les personnes en présence reconnaissent ce qui autorise ce personnage à officier et à recevoir un tel paiement - le brahmane est à leurs yeux le garant du rite -, tandis que dans l'autre (celui du patuā), ce n'est pas aussi clair. Même en tenant compte de l'influence que l'hindouisme peut avoir sur les Santals, le fait que le patuã "représente" le brahmane

17 Il faudrait pour cela aussi bien connaître le monde hindou que le monde santal. Un certain nombre d'auteurs pensent par ailleurs que les patuā auraient pu faire partie de ces groupes qui ont été fortement influencés par le bouddhisme avant d'être (pour une partie d'entre eux) convertis à l'islam. Cette pratique aurait-elle des attaches bouddhistes? On ne le saura sans doute jamais.

${ }^{18}$ C'est bien, semble-t-il, une des forces du système indien que de faire en sorte que l'autorité rituelle (dès lors qu'elle s'exerce dans le domaine public au moins) soit toujours "brahmanique". Notons au passsage combien la pensée hindoue est attentive à la plus "humble" des activités (les jädu patuã sont aussi peu nombreux que pauvres et leur activité reste somme toute assez limitée), cherchant toujours à intégrer. Quel rôle joue le patuă dans cette intégration? C'est sur cette question que J. B. Faivre nous laisse à la fin de son article. Il voudrait "mieux comprendre comment a pu s'opérer cette étonnante fusion - ou simplement interprétation - d'éléments de mondes religieux si différents (santal, hindou et plus exceptionnellement musulman). Ceci permettrait de juger s'il s'agit d'une confusion obtenue par les jädu patuã pour mieux arriver à leurs fins $[\ldots \mid$ bref de tâcher de savoir si ce jeu d'associations qu'ils ont inventées ne prête pour eux à aucune conséquence" ? (Faivre, 1980: 120). 
(comme dit J. B. Faivre) peut-il suffire à leur faire admettre que ses actes soient efficaces?

\section{Des "images" et un "peintre" pas comme les autres}

Revenons sur les termes pat et patuã. Le mot pat est, nous l'avons vu, habituellement traduit par "peinture". Mais il ne se limite pas à ce sens. Le terme s'applique en effet au Bengale, dans le langage courant, non seulement à ce que nous appelons des "peintures" (sur toile ou sur papier, plus rarement sur bois ou sur d'autres matériaux) mais aussi à des stèles (de pierre) ou à des plaques (de métal ou de bois) qui, tout en comportant une image (parfois en relief), ne sont pas nécessairement peintes (les plaques de métal en particulier ne sont pas peintes). Dans certains cas, il est difficile de dire si le terme pat désigne la représentation imagée, son support, ou bien les deux à la fois, comme si, en ce pat, l'idée d'image n'était pas dissociable, ou dissociée, de celle de son support. On accepte généralement que pat vient d'un nom sanscrit: pata (racine verbale pat, "bouger, déchirer"), dont l'un des premiers sens est "tissu; bande étroite de tissu" (angl.: woven cloth, cloth, narrow piece of cloth", Monnier-Williams, 1963). C'est aussi un des sens premiers de pat en bengali, et, dans les dictionnaires bengalis anciens, le patuā apparaît d'abord comme tisserand (sens perdu aujourd'hui) (articles pat et patuā dans Haughton, 1833, et Mitra, 1906) ${ }^{19}$. L'idée de tissu - mais aussi celle de bande - est donc première par rapport à celle de peinture. Si cette idée s'est beaucoup affaiblie dans l'usage courant du mot (qui tend de plus en plus à désigner une "image"), celle de support semble en revanche

19 L'enquête ethnographique montre que les patua du Bengale ont encore sous différentes formes des relations avec le tissage. Ils ont des liens étroits avec certaines castes de tisserands musulmans. Par ailleurs, c'est chez les patua qu'on se procure le fil dit "ghunsi" posé rituellement aux poignets des enfants peu après leur naissance. Dans le temps, ce fil n'était pas, semble-t-il, fait de coton, mais de pãt ou chanvre. Dans les régions de culture de ce végétal, le travail du chanvre revenait à des groupes appelés "patwa"(ou pãtwa ?) qui pratiquaient également la peinture. 
encore très présente. Par cette référence au support, la notion de pat se différencie de celle de citra (racine verbale sanscrite: cit, "percevoir"), autre terme bengali également traduit par "image" et "peinture", mais qui renvoie à l'idée de lumière, de clarté, liée à celle de variété, de multiplicité d'impressions.

Le terme patuā, nous l'avons vu, a pour sens premier: "qui a un rapport au pat". Il renvoie d'abord à un individu. Mais on parle également de "patuã jāti". On connaît le sens initial de "naissance" (plus précisément: "être né") qu'a ce dernier terme, ainsi que les deux principales traductions qu'il reçoit selon le contexte où il est utilisé, à savoir tantôt "espèce", tantôt "caste", ce deuxième sens ayant plus particulièrement retenu l'attention des ethnologues. Qu'en est-il dans le cas qui nous occupe? Un examen approfondi de ces questions dépasserait le cadre de ce travail. Nous noterons seulement ici que, au Bengale: 1) le terme patuā désigne aussi bien des individus que des groupes; 2) de tout patuā on peut dire qu'il est "jăti patuă"; 3) tous les individus recevant le nom de patuā ne font pas nécessairement partie de la caste ${ }^{20}$ de ce nom (il y a sur ce dernier point une différence notable avec ce qui se passe habituellement pour les autres castes: un individu appelé kumbhakär par exemple, "faiseur de pots", fait nécessairement partie de la caste du même nom). C'est ainsi que l'on trouve parmi diverses castes des individus voire des "sous-castes" entieres désignés comme patuã. C'est notamment le cas chez les "potiers" ou kumbhakar, les "charpentiers" ou sūtradhar, mais aussi dans certaines castes de brahmanes (considérées il est vrai comme "dégradés") ${ }^{21}$.

Il faut donc distinguer au Bengale entre 1) les patuã qui relèvent de castes variées (Sütradhar, Kumbhakar, etc.) et 2) les patuã qui relèvent de la caste qui porte ce nom ${ }^{22}$ (nous les appellerons "patuă

${ }^{20}$ Le terme caste est entendu ici au sens de groupe fondé sur un principe d'endogamie, ayant un statut évalué en fonction de la naissance (plus ou moins pure).

${ }^{21} \mathrm{Ce}$ qui est dit ici vaut généralement aussi pour le terme citrakar. Ni l'appellation patıă, ni l'appellation citrakar ne sont done suffisantes pour déterminer la caste de la personne ainsi désignée.

22 G. Dutt est l'un des sculs auteurs à rappeler cette différence. Mais il ne se demande pas ce qui permet de réunir ces deux types de patuā sous une même appellation. 
de caste"). Dans ces deux cas, le rapport du patuā au pat est très différent. Dans le premier cas, le pat fabriqué est destiné à un tiers, qu'il soit vendu (dans les lieux de pelerinages, les foires, etc..) ou exécuté sur commande, dans un but rituel ou non. Il peut s'agir de toiles peintes, d'illustrations de manuscrits, de peintures murales, de décors de spectacle, etc... Le support ou la taille du pat est éminemment variable, mais il a presque toujours la forme d'un panneau (carré, rectangulaire, circulaire...). Autre caractéristique: quand il est exposé, il est visible d'un seul coup d'œil dans son ensemble. Lorsqu'il est le support d'un culte, il représente généralement une divinité, dessinée seule, ou entourée de diverses petites scènes; la divinité occupe alors le centre ou l'apex du panneau, les autres éléments imagés étant répartis sur le pourtour. Il faut remarquer que ce type de peinture est aussi bien désignée par le terme citra que par le terme pat, les deux mots semblant dans ce cas se recouvrir parfaitement. En reprenant une catégorie souvent utilisée par les indianistes, on peut dire que, pour ce premier type de pat, le rôle du patua - son "rapport au pat" - se limite à celui d'un fabriquant (beng. kar, "celui qui fait").

Dans le cas du patuā "de caste", ce qui constitue le "rapport" du patuă au pat est tout autre. Les pat dont ces patuā sont les spécialistes se présentent sous la forme de rouleaux, raison pour laquelle on les appelle aussi, pour les distinguer des premiers, jarāno pat, "pat enroules" ${ }^{\text {23 }}$. Pouvant mesurer jusqu'à 10 mètres de long pour seulement 20 à $50 \mathrm{~cm}$ de large, ils comportent une succession de petits tableaux disposés en ordre vertical, le tout semblant former une histoire. Au début et à la fin du rouleau, un tableau de plus grande taille que les autres représente une divinité, qui donne son nom au pat. Fait pour être transporté, ce type de pat est toujours gardé enroulé, l'ensemble étant protégé par un tissu. Le pat lui-même est exécuté sur papier ${ }^{24}$. Normalement, ces pat ne sont pas vendus. Ils sont utilisés

23 Les pat de type "panneau" sont parfois appelés caukoś pat, "pat à 4 côtés". Caukoś et jarāno renvoient bien à des catégories. Certains caukós pat sont en effet gardés enroulés lorsqu'ils ne sont pas exposés. Les patuā "de caste" font aussi des pat de type "panneau" mais l'activité qui les marque en propre est le rouleau-peint.

24 Il y a quelques exceptions, contemporaines. 
par le patuā pour être montrés de porte en porte chez les particuliers, accompagnés de la récitation d'un texte. Au moment de la présentation, le patuã entonne le récit correspondant au pat qu'il va montrer; en même temps, il ouvre le rouleau sur la première image; il le déroule ensuite de bas en haut au rythme de ses paroles, ne montrant jamais qu'une seule image à la fois. Le pat n'est donc jamais exposé dans son ensemble, et il est toujours accompagné de l'énonciation d'un texte, dit par le patuã.

A la différence du pat de type "panneau", on dira de ce type de peinture qu'elle "a un grand nombre de citra" (ei bohu citrer pat: c'est un pat avec beaucoup d'images); mais elle ne pourra recevoir l'appellation de citra. Elle comporte des citra mais ne peut pas être citra. La pluralité des images est ici une nécessité intrinsèque de l'objet (dans le pat-panneau, il n'y avait qu'une seule image; ou, lorsqu'il y en avait plusieurs, c'est comme si ces différentes scènes n'étaient que les multiples reflets, ou impressions, d'une même manifestation, représentée au centre du panneau). Autre différence: le pat-panneau se lisait dans n'importe quel sens, et il ne s'y attachait aucune idee de continuité, tandis que le rouleau-peint du Bengale se lit nécessairement dans la succession.

Le terme pat ne recouvre donc pas tout-à-fait celui de citra. Nous ferons sur ce sujet une dernière remarque. Aux notions de pat et de citra peuvent être associées aussi bien celles de lekhā, "écrire" que de ankā "dessiner"25. On trouve en effet pat (et citra) ankā, pat (et citra) lekhă. Toutefois, dès lors qu'il s'agit de rouleau-peint, nous avons constaté que seul lekhā est utilisé; les patuā ne disent guère pour leurs rouleaux: pat ankā, alors qu'ils utilisent couramment l'expression pour d'autres formes de pat. Autrement dit, le rouleau-peint "s'écrit" mais ne se "dessine" pas (on peut certes "dessiner" les différentes

25 Ankã (verbe et nom) est dérivé de ank, "marque", puis "ligne". On le traduit par "dessiner", "écrire", "délimiter". Singha (1957: 17) lui donne aussi le sens de "modelé" (en Inde, la sculpture est penséc comme prenant idéalement naissance dans le dessin, ce dernier étant conçu, et effectivement encore aujourd'hui réalisé par la plupart des artisans, à partir du point, situé idéalement au centre du dessin. Le terme ankā renverrait donc à l'idée d'un espace développé à partir du point). 
images qui le composent, mais l'ensemble des scènes qui le constituent ne peut être qu'une "ériture").

Dans le cas du patuã de caste, la vision de la gloire divine que procure l'écoute et la vue du pat est, nous l'avons dit, intimement liée à son déroulement. Dans ces chants et ces images, la notion de chronologie n'est pas pour autant nécessairement présente. L'analyse du corpus des chants patuā montre en effet qu'un pat peut avoir une faible structure narrative, ne faisant que des allusions à des épisodes d'histoires connues de tous, et certains pat sont totalement dénués "d'histoire". L'idée d'une succession d'actes venant s'ajouter les uns aux autres pour donner un résultat semble donc ce qui prime. Peut-on aller jusqu'à distinguer, au Bengale, un patuā-"écrivain", qui serait lié au temps, d'un citrakar-dessinateur, qui serait lié a l'espace ? Toujours est-il que le patuã de caste se présente bien davantage comme un "écrivain" qui consigne des séries d'actes-événements, que comme un dessinateur qui développe un thème dans un espace ${ }^{26}$. L'analogie est forte avec le personnage bien connu de Citragupta, scribe assigné, selon la tradition hindoue, à tenir registre des actes qui se sont succédés dans la vie des mortels afin d'en rendre compte en temps voulu à Yama le dieu des morts. Ce lien entre Yama et le personnage du scribe, en lequel la plupart des auteurs voient une fonction (l'écriture est considérée comme l'une des fonctions de Yama) Ch. Malamoud le fait apparaître comme un trait constitutif de la nature de Yama: "par association à Citragupta, scribe chargé de noter les faits et gestes de chaque mortel [...] Yama apparaît, à partir des Puranas, comme un dieu d'écriture" (1989: 126). Et il a cette formule qui vient singulièrement éclairer les matériaux bengalis que nous présentons ici: "Symétriquement, l'écriture est du domaine de Yama et porte les signes de la mort" 27 .

26 Il serait intéressant d'approfondir sous eet angle les rapports qu'entretiennent dans la pensée hindoue le dessin et l'écriture. 11 n'y a évidemment pas opposition entre les deux, comme le montre le lien existant entre Citragupta et Yama (cf. ci-après). Certains textes classiques indiquent que le dessin $(a n k a ̈)$ est englobé dans l'écriture (lekhā).

27 On retrouve l'idée de "porter" dans patidär (un des noms du patuã), "celui qui porte, celui qui a la charge" du pat. 
Dans ce même texte, qui porte sur le scribe (lekhaka) royal, plus précisément sur la relation entre l'écriture et la fonction royale, Ch. Malamoud fait référence à un personnage que mentionnent souvent les publications sur les patuā du Bengale, et plusieurs auteurs qui ont travaille sur la peinture indienne: il s'agit du yamapattikā. On reconnait dans cette appellation le nom de Yama et le terme pat; il s'agit bien de mort (ou tout au moins du dieu des morts) et de représentation imagée. Or, que fait ce yamapattikā ? Précisément, il montre des rouleaux-peints. Se référant à deux textes sanscrits anciens ${ }^{28}$, Ch. Malamoud écrit: le yamapattikā commente des "histoires peintes sur un rouleau [... où] sont figurées des scènes du jugement des morts. Les personnages principaux sont évidemment Yama et son scribe secrétaire Citragupta". Le yamapattika est décrit exactement dans les mêmes termes dans le Harșācaritā de Bāna (chap. V: 136), texte sanscrit daté des VIIe - Xe s. ${ }^{29}$ auquel se réferrent généralement les auteurs signalés plus haut. Peut-on pour autant, comme certains l'ont écrit, considérer que les patuă "de caste" seraient des "descendants" du yamapattika $\bar{a}^{30}$ ? L'éloignement dans le temps et dans l'espace

28 Il s'agit: 1) du Mudraraksasanakakatha de Mahadeva (trad. de S. Piano en italien, 1968), adaptation tardive du Mudraraksasanakakatha daté entre le IIIè et le IXè siècle de notre ère (Renou, Inde classique, t. II.); et 2) du Nitivakyamrita de Somadeva Suri (trad. de O. Botto en italien, 1963), texte écrit par un jain digambara vers 959 de notre ère, peut-être dans l'état de Hyderabad. La consultation de ces textes montre que, dans le second, le yamapattika apparaît dans la liste des car, terme que le traducteur italien rend par le spie, "espion", notion plus particulièrement développée par $\mathrm{Ch}$. Malamoud qui note que le yamapattika appartient aux "métiers qui servent de 'couverture' aux espions envoyés par le roi". Monnier-Williams (op. cit.) indique pour sa part que le car est "celui qui bouge". Nous reviendrons sur ces points.

29 Nous avons consulté ce texte dans la traduction anglaise de Howell, 1897. On pourrait s'étonner de retrouver dans plusieurs textes sanscrits presque mot pour mot la même description du yamapattika. Que les auteurs sanscrits se soient mutuellement copiés ou non, cela montre à notre sens que le yāmāpattikã était déjà, à cette époque, un "personnage".

30 Il est intéressant de noter que ce "raccourci", qui tend à évacuer aussi bien les faits historiques que les réalités sociales, est le fait d'auteurs indiens. Notons qu'il existe en Inde dautres groupes qui, par leur activité et/ou par leur nom, peuvent être rapprochés du yamapattika. Il y a entre eux et les patuã du Bengale des différences notables. 
interdit une telle conclusion. Rien n’indique avec certitude que le Bengale ancien ait connu de tels yamapattikä. encore moins que ceuxci aient, comme de nos jours au Bengale, formé une caste. Le rapprochement mérite pourtant d'être fait. et c'est sans doute ce que veulent faire entendre ces auteurs. Mais sur quel plan? Ch. Malamoud limite résolument la discussion au niveau des représentations. "Le yamapattikā est dans la familiarité constante de Yama" écrit-il.

Au regard de cette définition - qui sied étonnamment à notre "peintre-magicien" - les faits bengalis restent cependant complexes. En effet, seuls certains patua $\bar{a}^{31}$ - précisément ceux que l'on appelle jādu patuā - sont considérés comme des spécialistes de ce qu'on peut appeler l'écriture funèbre, alors que tous les patuã "de caste", nous allons le voir, commentent des "histoires peintes sur un rouleau [...] où sont figurées des scènes du jugement des morts [... avec] Yama et son scribe secrétaire Citragupta" pour reprendre les termes de Ch. Malamoud. Expliquons-nous. Au Bengale, les patuã "de caste" ne se présentent pas comme un ensemble simple. On y distingue les patuă proprement dits et les jadu patua $\bar{a}^{32}$. Il s'agit là de deux groupes distincts, qui occupent des aires géographiques voisines, aux caractères marqués, et que les Bengalis opposent: d'une part les terres alluvionnaires du Gange comprises entre les collines de l'ouest et la rivière Bhāgirati (traditionnelle limite entre le Bengale occidental et le Bengale oriental), d'autre part cette zone de collines et de "forêts" habitée par les Santals que nous avons décrite plus haut. Il n'y a en principe pas d'intermariage entre les deux groupes; et tandis que les patuã que nous pouvons appeler "de la plaine" sont en majorité musulmans, ceux des "collines" seraient plutôt hindous ${ }^{33}$. Chaque groupe utilise un type de

31 Rappelons qu'il ne s'agit ici que des patuă "de caste", les seuls à montrer des rouleaux-peints.

32 Nous avons mené des enquêtes dans les deux groupes, l'essentiel de nos recherches ayant toutefois porté sur les premiers (les patuā proprement dit).

${ }^{33}$ Les patuã "de caste", particulièrement les jädu patuā, sont très mal connus. Pour des raisons diverses (nous en évoquerons certaines plus loin) qui ne tiennent pas seulement à leur faiblesse numérique (ils seraient, pour les deux groupes confondus, environ 10000 à 15000 au Bengale), ils n'ont jamais été correctement recensés, même à l'époque de l’administration anglaise, pourtant très diligente en la matière. 
pat bien défini. Les patua de "la plaine" font des rouleaux-peints de grande taille, d'un style pictural relativement élaboré, représentant des themes de la mythologie hindoue (dont le yampat, représentation de Yama et de son royaume). Ils visitent surtout les foyers hindous. Les patuā "des collines" (jādu patuā) font quant à eux deux types de pat: les pat du premier type sont très semblables par leur forme, leurs themes ${ }^{34}$ et l'utilisation qu'en font les jādu patuã, à celui des patuā "des plaines"; mais ils en different par la taille, le style pictural, et la "clientèle". Celle-ci est aussi bien hindoue que santale (ou musulmane), les mêmes rouleaux étant présentés aux différentes communautés avec un accompagnement oral différent (d'une même image, le patuā peut faire plusieurs "lectures" en fonction de la situation rencontrée). Le second type de pat utilisé par les patuā "des collines" dépasse rarement la grandeur d'une main. Bien que toujours "enroulés", ces pat comportent cette fois plusieurs séries d'images isolées ou assemblées par groupe de deux ou trois, chacune représentant un personnage unique, homme, femme ou enfant, entouré de quelques objets. C'est, on l'aura reconnu, les pat que nous décrivons ici. Ce sont ces pat, et la pratique qui leur est liee, qui font toute la différence entre patuā et jādu patuã. Les Bengalis sont unanimes sur ce point: les jādu patua "sont différents car ils montrent des images de mort(s) (mrtu pat)". Autrement dit les uns sont associés à la mort, les autres non ${ }^{35}$.

Il est intéressant de s'arrêter un instant sur la façon dont les Bengalis ont traité la différence entre patuã et jädu patuā, tout en sachant que l'examen de cette question implique une réflexion approfondie - que nous ne pouvons entreprendre ici - sur la façon dont s'organisent autour de la notion de jāti des faits sociaux et des traits

${ }^{34}$ Certains de ces rouleaux portent sur la mythologie santale, d'autres reprennent des thèmes hindous. Parmi eux, on retrouve les yampat.

35 Les patuā "des plaines" seraient plutôt assuciés à la fécondité. Ceci ne serait cependant pas en contradiction avec l'idée que le patuā "de caste", quel qu'il soit, est un "familier" de Yama. W. Wilkins en effet (1975: 83) dit que les filles non mariées rendent un culte à Yama pour qu'il leur procure un mari. Ce dieu est donc associé, sinon à la fécondité, du moins au mariage. On sait également que c'est l'accouplement (jamais réalisé) de Yãma avec sa sœur jumelle Yämi qui a donné naissance aux hommes (Malamoud, 1991). 
liés à l'activité des groupes considérés. Patuā et jādu patuā sont généralement désignés par les termes génériques de patuã (ou citra$k a r)$. On peut néanmoins les distinguer lorsque le besoin s'en fait sentir en ajoutant au terme patua a (et seulement à ce terme, celui de citrakar n'étant pas utilisé dans ce cas) diverses épithètes. Seuls les patuă "des collines" sont concernés par ce traitement. Ils reçoivent les noms de jangli patuā (patuă "forestier"), sāntâli patuã (patuā "santal"), ou encore, nous l'avons vu, jādu patuã (patuã "magicien"), ces locutions étant, localement, souvent abrégées en jangli, sāntāl ou jādo ("de la forêt", "santal", "magicien"). Les patuã de la plaine, quant à eux, sont seulement désignés par le terme patua $\bar{a}^{36}$ (la plaine semblant donc constituer la référence).

Avoir un rapport avec ce qui est de la "forêt" et "santal" est donc, comme la "magie", ce qui distingue le jādu patuā des autres patuā, au moins au niveau des désignations. On pourrait sans doute comprendre, grâce aux récents travaux indianistes, ce que recouvre l'opposition entre les termes "forêt", "tribu", "santal" d'une part, et "village" et hindou (ou appartenant à la société des castes, les patua des plaines étant reconnus comme tels) d'autre part. Il est moins aisé de dire comment la "magie" se place dans cet ensemble de notions: en quoi un patuã des collines s'oppose en ce domaine à un patuã de la plaine. Il est sûr qu'on ne peut s'en tenir ici à une opposition simple de type rite/magie (la magie occupant le pôle inférieur, celui du jädu patuã, et le rite - qu'incarne le brahmane - le pôle supérieur). On sait en effet, notamment par les études de W. Calland et $\mathrm{L}$. Renou, que le brahmane, spécialiste du rite, est aussi en Inde, dans bien des contextes, le dépositaire d'un savoir magique. Nous ne pouvons entrer dans ce vaste domaine ${ }^{37}$; nous aborderons seulement la question du $j a ̄ d u$ dans le cadre qui est le nôtre. Il faut préciser que les Santals, comme bien des hindous de basse caste, désignent habituellement le

${ }^{36}$ Dans le district de Medinipore, ils reçoivent toutefois également le nom de duäri patuāa, "patuā du seuil" ou "de la maison".

${ }^{37}$ On peut toutefois noter que l'art magique du brahmane n'est pas qualifié de jãdu. 
jādu patuã par le terme jâdo ${ }^{38}$. La notion "d'écriture" - et les connotations mortuaires qu'elle peut comporter - est donc absente des représentations qu'ils se font de ce personnage. Pour les Santals, le jādu patuā n'est ni peintre ni scribe. Les forces qu'il mâttrise relèvent du $j a d o^{39}$. Nous comprendrons peut-être mieux pourquoi au terme de l'exposé qui va suivre.

Le patuā, le mort et le survivant. Extraits de nos carnets de terrain.

Nous avons vu que le jādu patuā n'est pas santal et qu'il réside dans des quartiers séparés, voire assez éloignés des villages santals. Il est néanmoins fort au courant de ce qui s'y passe. C'est ici qu'intervient un trait notable du personnage: le patuā est toujours en mouvement, passant une part importante de sa vie sur les chemins, en "transit" donc, un peu à l'image de ce défunt dont il porte l'image et dont il récupère les habits. Cette mouvance, il la partage avec la plupart des membres de sa caste, hommes, femmes et enfants y pratiquant diverses activités de colportage. Contrairement au colporteur cependant, le montreur de rouleau ne vend rien. Sa manière de faire differe aussi de celle de ce dernier. Ainsi, quand il s'éloigne de chez lui pour montrer des pat, c'est toujours en solitaire. Nul ne sait où il va et il semble garder jalousement le secret de ses allées et venues.

Ce goût du secret semble être chez lui comme une seconde nature, qu'il manifeste aussi bien vis-à-vis des siens qu'à l'extérieur. Tous les auteurs ont noté la capacité du patuā à éluder les questions qui concer-

38 Les termes de patuã et citrakar ne sont pas inconnus des Santals, mais ils les utilisent essentiellement en contexte hindou, avec le sens d'un nom propre. Jädo est un mot bengali. Le jädo est différent de l'ojhä, sorte de prêtre à la fois devin, guérisseur et exorciste qui exerce aussi bien chez. les Santals que dans les basses castes hindoues et musulmanes, groupes dont lui-même provient. Certains patuã sont des ojhâ réputés, mais leur fonction en tant que tel ne se confond jamais avec celle qu'ils ont en tant que montreurs de pat.

39 Rappelons que la société santale était "sans écriture" jusqu'à une date récente (c'était aussi le cas des basses castes). Les représentations figuratives y étaient par ailleurs très peu nombreuses, ct extrêmement schématiques. 
naient certains domaines de son activité (ce qui ne veut pas dire qu'il ne puisse être par ailleurs excessivement affable, au contraire même). Il n'est pas toujours aisé de l'approcher; il est particulièrement méfiant envers les bhadra lok (gens de bonnes manières, gens éduqués) et tout ce qui ressemble de près ou de loin à un officiel ou à un notable ${ }^{40}$. Effectuant des tournées avec eux, nous avons maintes fois constaté qu'il révèle rarement d'emblée sa véritable identité. Son art de l'esquive en ce domaine est remarquable et il préfere mentir effrontément plutôt que de "s'exécuter". C'est également à cette occasion que nous avons appris que les patuã ne montrent pas leurs pat dans les lieux publics. Bien au contraire, quand ils se déplacent, ils les tiennent serrés sous leur bras, cachés sous leur chemise ou sous le châle dont ils s'entourent souvent les épaules, et bien malin qui pourrait en deviner la présence. C'est spécialement vrai pour le jădu patuā, dont les pat sont toujours de petite taille, et nous allons bientôt comprendre pourquoi.

A ces manières un peu particulières fait pendant le silence dont ses principaux "clients" entourent le personnage et ses activités. Nous avons déja signalé que les Santals cherchent à éviter la venue du jadu patu $\bar{a}^{41}$. Si ce dernier vient tout de même à se présenter, ils peuvent semble-t-il l'accueillir avec égards (M. Archer, 1977, texte cité), mais ils taisent soigneusement le fait. Ainsi des Santals que je pensais bien connaître ont toujours nié recevoir la visite du patuã jusqu'au jour où, par recoupements, je compris qu'ils me mystifiaient. A partir de ce moment, le silence se brisa, et je devins au contraire pour eux une mine de renseignements utiles, voire une sorte de protection. La visite

\footnotetext{
${ }^{40}$ Les campements de jâdu paluā sont souvent éloignés des centres de communications. Certains ne sont accessibles qu'à vélo ou à pied, et l'on n'est jamais sûr que ses habitants ne se soient pas évaporés à l'annonce d'une arrivée inhabituelle. Un jour que, non encore connue des jädu patıã, et peut-être trop bien accompagnée, j'avais enfin réussi à rejoindre l'un de ces campements, tous les hommes en étaient partis le matin même. J'appris ultérieurement qu'ils avaient fui à l'annonce de notre venue.

41 Je me souviens encore d'un Santal qui avait eu un deuil dans son village et avait aperçu sous un arbre un campement qu'il soupçonnait être de jädu paluă. Sachant que j'avais des contacts avec ces gens peu recommandables, il me supplia de ne rien laisser filtrer de ce qui se passait chez lui.
} 
du patuã est et se veut donc sans trace ${ }^{42}$. Quant au personnage luimême, il nous fait plus que jamais penser à ce yamapattikã des temps anciens: à la fois "celui qui bouge" et "l'espion" (Monnier-Williams, 1964; Malamoud, 1989).

Si à travers ses déplacements et ceux de ses proches, le patuã a de multiples occasions de se tenir au courant des affaires villageoises, son lieu d'espionnage favori reste l'échoppe de thé. Car son projet se réalise dans la plus grande discrétion, mais prend naissance dans la rumeur publique. Tous ceux qui ont voyagé en Inde connaissent ces échoppes des bords de route: quelques piquets de bois mal taillés soutenant des panneaux de palme tressée ou des vieilles toiles de sac savamment disposés de manière à apporter, à toute heure de la journée, le maximum d'ombre, un foyer toujours rougeoyant, deux ou trois bancs de bois, un présentoir abritant quelques gâteaux qui attendent un voyageur peu regardant, c'est là le domaine du chá-wāla, le "chargé au thé", qui, inlassablement, sert des petits verres de the au lait, sorte de breuvage à la couleur et au goût indéterminés ${ }^{43}$. Au long des heures qui s'écoulent dans l'attente, toujours incertaine, d'un bruit de moteur qui indiquera que le bus de ligne n'est pas en panne ce jour-là, la conversation suit le cours des événements locaux.

- "Hier, untel est mort..." - "Avait-il des enfants ?... Comment est-il mort ?.." - "Quel âge avait-il ?"

- "Son fils aîné ne s'appelait-il pas Ram ?"

- "C'était un homme aisé, il avait 2 vaches qui donnait un lait très fin, et de nombreux poulets... N'avait-il pas marié son dernier fils récemment ? Qui y a-t-il dans la maison maintenant ?"

Les uns avec compassion, les autres pleins de curiosité, certains par intérêt, chacun selon ses mobiles intervient, écoute ou pose des

42 Peut-être est-ce ce qui explique que les spécialistes des Santals parlent si peu des jădu patıå ? A moins que la pratique du patuã ne faisant pas partic des rites santals, ils ne s'y soient pas intéressés?

43 Dont le nom trouve sans doute une justification lorsque l'infatigable préposé, jugeant un client plus digne que de coutume (et aussi plus solvable), sert sans crier gare un "special", thé plus corsé et avec une double ration de lait, mais aussi plus onéreux! 
questions. Au rythme des petits verres de thé, les nouvelles circulent... sans que nul ne se soucie de l'identité des personnes présentes.

Petite scène banale et sans conséquences au détour d'une petite route du Bengale ? Elle le serait pour beaucoup. Pas pour tous cependant, et en tout cas pas pour le jādu patuã. Pour lui, elle est au contraire d'un grand intérêt, et s'il s'en trouve un là par hasard, tous ses sens sont en éveil, bien qu'il n'en laisse rien paraître, comme le constatait un informateur: "Si le jādu patuă apprend que quelqu'un est mort dans une famille santale, il se rend dans le secteur. Les gens ne savent pas [qu'il est là; et lui] rien qu'en restant assis à la boutique de the, il apprend tout ce dont il a besoin". Certains ajoutent, d'un ton qui en dit long sur la réserve qu'ils observent à l'égard de ce personnage et de ses attributions "magiques": "il n'y a rien de difficile à cela".

Bien plus tard un patuã (de la plaine) que je connaissais depuis plusieurs années vint, au détour d'une conversation portant sur tout autre chose, confirmer cette façon de faire, continuant par un récit qui livra peu apress un véritable texte - celui que je présente plus bas. Il conmença ainsi: "un jour, je suis allé chez les Santals (c'est à dire en réalité: je suis allé chez les jādu patuã qui visitent les Santals)... Je leur ai montré mes pat, et puis, à l'un d'eux, j'ai demandé: 'Et toi, comment gagnes-tu [ta vie] ?' Il me dit: 'Frère, viens avec moi, je te montrerai' ${ }^{44}$. Il m'emmena avec lui. En chemin, je le voyais qui s'arrêtait souvent; à l'un, il demandait du feu, à l'autre, il offrait un verre de thé... Il disait: 'j'ai appris qu'un tel est mort...' et ainsi, l'air de rien, il venait à savoir des tas de choses. Soudain, il demande: 'comment s'appelle le fils aîné ?'".

La soudaine précision de ces derniers mots nous a longtemps laissée perplexe. Et pourquoi ce subit passage au présent ? C'est bien plus tard, en comprenant la place que tient le fils aîné dans le travail de deuil, que nous avons saisi toute l'importance de cette question,

44 Il ne faut pas se tromper sur la valeur de ce récit. Au-delà de son caractère anectodique, qui est réel, une phrase comme: "un jour, je suis allé chez les Santals" rappelle notre "Il était une fois", comme si, au-delà de ce patuā particulier, c'était son groupe entier qui parle. Ce groupe avait d'ailleurs, nous le savions, des relations régulières et anciennes avec les jädu patuã. 
compris aussi que le patuä nous restituait là quelque chose d'essentiel. Le patuã continua en décrivant l'attitude et les paroles prononcées par le jādu patuă lorsqu'il se présenta devant la maison du défunt. Cette recension est exceptionnelle à plus d'un titre. Tout s'est passé comme si, fin connaisseur de ces sortes de cousins que sont pour lui les jadu patua, notre narrateur avait condensé là l'essentiel de la pratique. Les mots, les expressions importantes, le rythme du jādu patuā, sont là. C'est du moins ce qui apparait lorsqu'on compare ce témoignage avec d'autres, provenant cette fois de jādu patuā eux-mêmes (mais que nous n'avons pas retenus parce qu'ils étaient encombrés d'éléments ésotériques exprimant des idées ou des doctrines religieuses particulières - sufi, baül ou autres - très répandues dans le Bengale rural, mais qui viennent inutilement compliquer la compréhension du texte) ${ }^{45}$.

C'est donc un exemple simple que nous présentons. Nous pensons, nous l'avons dit, qu'il s'agit d'un véritable texte. L'énonciation commence et se termine par des formules précises. Nous le restituons dans sa presque intégralité, en serrant la formulation bengalie, car c'est en s'attachant de près au texte et à sa forme, notamment à sa forme grammaticale, que l'on peut rendre quelque chose du travail du patuã. Certaines tournures de langue, des "erreurs" de prononciation, des distorsions de mots, des répétitions, souvent considérées par les Bengalis un tant soit peu éduqués comme des imperfections ${ }^{46}$, l'apparence d'une certaine confusion enfin, mais un ordre saisissant dès lors que l'on reprend le récit phrase après phrase, un ordonnancement, un

45 Nous avons résidé longtemps en milieu rural mais n'avons jamais pu observer la présentation des pat mortuaires "in situ". Lorsqu'on relit les meilleurs auteurs sur la question, on s'aperçoit qu'il est impossible de dire si pour leur part, ils avaient euxmêmes observé la scène, ou bien s'ils basaient leurs remarques sur des récits de tiers (patuã ou autres) et leur connaissance d'un milieu. Le doute est d'autant plus justifié lorsqu'on connaît les habitudes des patuā d'une part, celles des administrateurs d'autre part (qui recueillaient le plus souvent leurs informations auprès de tiers)

${ }^{46}$ Rappelons-nous que le jādupatuā est généralement illettré. Il s'exprime dans une langue populaire, où les formes dialectales sont nombreuses. Au bengali, il mélange par ailleurs souvent des expressions ou des tournures hindi ou santali, le tout formant un ensemble qui écorcherait les oreilles de tout Bengali bien né. 
dosage extrêmement précis des éléments qui le composent, c'est ce type de choses que nous voulons soumettre à l'attention du lecteur. Nous donnons d'abord le récit en son entier, puis nous le reprenons phrase après phrase. Rappelons que ce texte est destiné à être entendu, et non lu sur une page écrite, c'est à dire que normalement, aucun retour en arrière n'est autorisé. Le rythme est rapide, la voix de celui qui l'énonce est très haut placée, les phrases, enfin, s'enchaînent sans marque de ponctuation.

Après avoir décrit comment le jādu patua s'était renseigné à droite et à gauche sur le défunt et sur son entourage, notre narrateur continua ainsi:

"il [le jādu patuā] se dit: 'celui-ci [le défunt] doit être ainsi; j'ai une image comme ça'. Ils [les jādu patuā] ont toujours sur eux beaucoup d'images (chobi) représentant des êtres humains; des images de Santals, ils en ont des centaines! Des garçons, des filles, des vieux, des jeunes, toutes sortes d'images, juste comme les Santals... S'étant renseigné sur son âge, il [le jādu patuā] s'assit un peu à l'écart, sortit l'image adéquate, puis se dirigea vers cette maison [celle du défunt] et se mit à héler, depuis la route, appelant le fils par son nom [le ton de notre narrateur change soudain; la voix se place un ton au-dessus. Il semble s'adresser à quelqu'un qui serait encore un peu loin. Ici commence manifestement le texte proprement dit]:

"Eh! apporte de l'eau, apporte de l'eau. C'est moi, ton pere, je suis là. Les jädu patuã l'ont dessiné (litt. l'ont fait se lever) sur l'un de leurs pat. Ton père arrive (à la maison)"... Il arrive [ou:] / j'arrive. Tu ne le crois pas; eh bien apporte de l'eau jaunie au curcuma et je montrerai". [Le ton redevient descriptif; le patuā parle de ce qu'il a vu]: "l'eau de curcuma fut apportée" Ipuis il reprend, avec le ton du jādu patuā]: "Mets-la dans une petite coupe. Non, ça ne va pas. Làbas se trouvent les grandes assiettes en kānsā (alliage métallique), apporte une de celles-là. Ton père m'a dit: apporte une de ces assiettes. Après l'avoir apportée, le "faire voir" aura lieu. Et ma poule jaune, elle est là ? - "Elle est là" - "Ta belle poule jaune, il m'a dit qu'il fallait la donner. Ton père m'a dit: donne une assiette, donne une 
belle poule, donne bien". [Descriptif]: "L'assiette et un peu d'eau de curcuma furent apportées. Ceci fait, il [le jadu patuā] montre un peu de (mot onomatopéique, presque incompréhensible) en disant: "Regarde ton pere et donne 5 roupies; donne 5 mesures de riz, donne une assiette, et les péchés qu'a commis ton père seront réduits... Le vieux m'a dit qu'il avait fait de la pomme de terre cette année; il y en a plusieurs kilos de remisés. Après avoir cultivé, ne doit-il pas manger lui aussi ? Il a peiné à travailler le sol et il est mort..."

Si nous reprenons maintenant le récit phrase après phrase, voici ce que nous y lisons. Il faut bien imaginer la scène. Nous sommes devant une maison de village. Les maisons du Bengale, particulièrement dans cette région de Medinipore d'où nous vient ce récit, ouvrent sur une cour, protégée des regards extérieurs par un mur assez haut. La vue est donc obstruée, mais l'ouïe ne l'est pas. Elle est même d'autant plus aiguisée que les yeux ne voient pas. L'appel lancé par le patuā est donc parfaitement entendu. Très vite (en l'occurrence, dès la phrase $n^{\circ} 2$ et en tous cas dès la phrase $n^{\circ} 3$ ), la famille du défunt comprend ce qui se passe, à savoir, que le jadu patuă est arrivé. N'empêche que quelque chose va se jouer comme si c'était le père qui était là. Tout se passe d'abord au niveau du seul discours.

De l'intérieur de la maison, on entend appeler:

1. " $X$ " (nom du fils du défunt) ${ }^{47}$

On ne sait pas encore qui c'est (le patuā ne s'est encore ni montré, ni déclaré) mais c'est sûrement quelqu'un de connu puisqu'il connaît le nom de celui qui habite la maison. Pour un villageois bengali, il est impossible de ne pas répondre à ce type d'interpellation. Si le fils est là, il répondra nécessairement, ou quelqu'un à sa place. Dans la maison, on imagine qu'il prête soudain l'oreille.

${ }^{47}$ Chez les Santals, comme chez les Hindous, le fils aîné est le deuilleur principal. 
2. "Eh! Apporte de l'eau, apporte de l'eau. C'est moi, ton père, qui arrive (jal niyāy, jal niyāy, ämi tor bābā esechi: litt. moi ton père je suis arrivé).

Celui qui s'exprime de cette manière ne peut être que le père, qui s'adresse à son fils. Si la désinence du verbe, qui correspond au $t u i$, 2 ème personne du singulier ${ }^{48}$, utilisée lorsqu'on parle à des personnes plus jeunes, à des inférieurs, ou a des familiers, ne suffisait pas à l'affirmer dans la première partie de la phrase, le "c'est moi, ton père" (ämi tor bābā) ne laisse plus aucun doute sur ce point.

Tout se passe donc comme si le perre revenait à la maison après une absence. Cette façon de s'exprimer correspond à un acte courant: dans les villages du Bengale, et c'est peut-être plus marquant encore dans les régions où l'habitat est dispersé, on a coutume de s'annoncer de l'extérieur. Les personnes auxquelles on doit un certain respect signalent plus particulièrement leur arrivée, en se désignant à la lère personne ("moi, j'arrive" ou "je suis là") ou à la 3eme personne (dans ce cas on aurait: bābă eseche, "père arrive"). La maisonnée ainsi prévenue, notamment les femmes, s'empresse pour accueillir l'arrivant. L'énoncé ci-dessus est une variante un peu plus élaborée de "moi, j'arrive", en ce sens qu'il met en scène également un "tu" ("c'est moi, ton père, qui arrive"), ce "tu" qui a été interpellé au début.

Dans un contexte ordinaire, l'eau demandée pourrait être celle que l'on apporte pour laver les mains ou les pieds de la personne qui arrive. La coutume est encore respectée dans de nombreux foyers ruraux, hindous comme santals. Avec cette eau, l'arrivant peut également se rincer la bouche. Dans le contexte où nous sommes, cette demande d'eau a peut être aussi une autre signification.

Il est aisé d'imaginer l'effet que peuvent avoir ces mots sur la maisonnée. Il peut être saisissant. Ce qui est certain en tout cas, c'est

48 Le bengali connaît 3 formes pour le pronom personnel singulier 2ème pers. Le tui marque l'affection ou la familiarité. Le tumi, qui est le plus usité, s'emploie entre personnes de même statut, de même âge, ou qui se connaissent bien. Le upni est honorifique. 
que, en dehors d'un contexte rituel (ce qui est bien le cas: du point de vue santal, aucun rite n'est en cours), personne, après la mort de quelqu'un, n'oserait prendre ainsi son intonation, encore moins prononcer les mots qu'il prononçait il y a peu.

3. "Les jädu patuã l'ont fait se lever sur l'un de leurs pat" (jädov patuã oder ektā pate uthiyeche)

Changement de perspective complet: le ton est le même, et l'on s'adresse toujours de l'extérieur vers l'intérieur de la maison, mais cette fois, ce n'est plus le père qui parle. C'est quelqu'un d'autre, un "il", qui donne une information sur deux autres "ils": le père et les jādu patuā. Ce "il" désigne ces personnes comme étant éloignées de la scène. En effet, on ne désigne pas à "la troisième personne" les personnes présentes. La forme employée pour le relatif: "oder" (2ème pers. pluriel) indique par ailleurs qu'il s'agit des jadu patuã (en général), et non d'un patuā particulier. Il n'est donc pour l'instant pas question de celui-là même qui se trouve à la porte (alors que c'est à cet instant que la famille comprend, sans doute possible, qu'il y en a précisément un là, énonciateur de ce discours). Par la construction de la phrase, on apprend que les deux absents de la scène (les patuã et le père) ont à faire ensemble; la flexion verbale nous dit que, dans cette action qui les lient l'un à l'autre, l'un est passif (c'est le père), l'autre est cause de l'action (ce sont les jādu patuā): le verbe otha est au causatif et a pour sujet les patuã.

Cette phrase peut être entendue à un premier niveau comme voulant dire simplement: les patuā ont dessiné ton père. En effet, quand l'art d'un dessinateur (et maintenant d'un photographe) fait apparaître une figure sur un support, on dit qu'elle s'y est "levée" (ou qu'il l'y a "levée"). Mais peut-être que le terme "lever" renvoie également à quelque chose d'autre. Cette phrase indique en tous cas, de manière indirecte mais claire, que le père n'est pas là. Par rapport aux deux premières phrases du discours, c'est une sorte de retour à la réalité en quelque sorte. 
Remarquons qu'en à peine trois phrases, le patuā a campé les trois personnages de l'action: le fils, le père, les jâdu patuă (et leurs pat), attribuant a chacun une "personne" précise: "tu", "je", "il". On débute par le "tu", qui représente le fils. Ceci n'est pas indifférent: c'est bien le fils qu'interpelle toute cette affaire. Est également donné le mouvement - déterminé par les variations de "personne" - qui va animer tout le texte. L'effet de surprise, soigneusement calculé par le jādu patuã (on comprend soudain pourquoi il est si discret dans son approche!) constitue le point de départ de cette dynamique. C'est un point essentiel que jamais le patuã ne néglige.

\section{4. "ton père arrive" (tor bāp āsche)}

Cette phrase peut être entendue de deux façons; deux personnes peuvent en effet s'exprimer ainsi:

a) le père, se désignant à la 3ème personne. Il s'annonce à nouveau à son fils comme s'il était là, tout près. Par rapport à la phrase précédente, il y aurait comme un retour en arrière: on revient à la position de la phrase 2.

b) une personne $X$, un "il", peut-être celui de la phrase 3; dans ce cas, le message serait: ton père s'est levé et il arrive (sur le pat); autrement dit, un patuā arrive.

Par rapport à la phrase précédente on observe que le temps du verbe change. L'emploi du présent (äsche) au lieu du passé composé (eseche), indique un mouvement: la présence (du père ou du patuă ? selon l'identité prêtée au sujet de l'énoncé) se fait plus proche, plus pressante. Cela peut avoir pour effet d'augmenter le trouble de celui qui écoute, tout en le pressant de prendre une position (notamment sur l'identité de celui qui s'exprime).

\section{5. "il arrive" ou "j'arrive" (āsche/āschi)}

L'ambiguïté continue et se complexifie. En un seul mot, le patuã offre quatre possibilités d'interprétations. En effet, la prononciation est ici telle qu'il est difficile de faire la distinction entre le " $i$ " et le "e", terminaisons verbales de la première et de la troisième personne du singulier présent. De surcroît, le patuā "mouille" légèrement cette voyelle finale, et cela d'autant plus aisément qu'elle est prononcée 
après une sifflante (ch). S'agit-il d'un "défaut" ? Est-ce voulu ? On peut se le demander. La rapidité de l'énoncé et le ton traînant - presque pleurnichard - que le jădu patuã adopte pour son discours accentue encore le phénomène. Notons ici l'importance de l'oral. Ce jeu ne pourrait exister à l'écrit. Enfin, notons "l'économie" de la méthode: un seul mot est prononcé.

- Premier énoncé possible: "il arrive". On retrouve les deux possibilités de la phrase précédente:

a) le père, parlant toujours de lui à la 3ème personne, confirme à son fils qu'il est là.

b) un tiers, confirme au fils que son père arrive bien.

- Le deuxième énoncé possible: "j'arrive" ouvre encore sur deux énonciateurs possibles du discours:

a) le père, se désignant cette fois à la lère personne, confirme qu'il est là.

b) le patuã, qui parlerait alors pour la première fois en son nom et a la lère personne, et manifesterait ainsi ouvertement sa présence.

Il est probable que c'est à ce moment que le patuã s'avance "à découvert"; ce qui suit montre en effet qu'à partir de ce moment - ou bien dès la phrase suivante - il a été reçu par le fils. La scène va à partir d'ici prendre une toute autre tournure. Jusqu'alors, le patuā, par une série d'ambiguïtés au niveau de la langue, avait crés puis entretenu le doute. Il va maintenant en quelque sorte lancer la balle dans l'autre camp. Il s'adresse au fils pour la deuxième fois; mais cette fois, c'est un défi: il laisse entendre que s'il y en a un qui crée le doute ici, ce n'est pas celui que l'on croît (à savoir le patuã ou le père), mais c'est lui, le fils. Autrement dit, le seul responsable dans toute cette histoire, c'est le fils. Et c'est à lui que revient de prendre une décision. Le patuã dit:

6. "tu ne le crois pas, eh bien apporte de l'eau jaunie au curcuma et je montrerai" (nā visvās karis, haldi jal niyāy dekhābo)

Si l'on se demande qui prononce la phrase, on pense d'abord au patuã (qui vient de manifester son identité). Le "je montrerai" qui suit 
semble mener à cette conclusion (remarquons toutefois que le patuā ne dit pas "je te montrerai" mais "je montrerai"). Mais ces mots peuvent également être attribués au père. Ils voudraient dire alors: "si tu ne crois que je suis là, je (te) montrerai (sous entendu: que je suis encore la)".

Deux cas se présentent donc. Cas $\mathrm{n}^{\circ} 1$ (celui qui vient d'abord à l'esprit): c'est le patuā qui parle. Une légère "irrégularite" de langue attire toutefois l'attention: c'est le "tui", 2ème personne du sg., réservée aux proches (marquée par les terminaisons is de karis et ay de niyāy) qui est utilisé ici, alors que normalement il devrait y avoir le "tumi", terme d'adresse normal entre personnes qui ne se connaissent pas (c'est ce qui se passe à la phrase 8 par ex.). Le patuã parle donc somme si il était le père ${ }^{49}$.

Cas $n^{\circ} 2$ : e'est le père qui parle. Dans ce cas, il annonce que, tout en étant mort, il peut cependant encore se montrer. C'est donc bien comme un revenant qu'il se désigne alors (ce qui change par rapport au début du discours, où, par un effet de surprise, le fils a pu avoir l'impression que son père revenait, c'est qu'ici le père se désigne luimême comme revenant. Il ne s'agit plus d'une "impression").

A ce niveau du discours, deux données nouvelles sont donc introduites par le patuã: le père se présente explicitement à son fils comme revenant; le patuã se présente comme pouvant parler au fils comme s'il était le père. L'énoncé comprend un autre élément important: il faut apporter de l'eau jaunie au curcuma ${ }^{50}$; cet acte est présenté comme pouvant seul lever le poids du doute dont le fils vient de se trouver malgré lui chargé (et l'enjeu est grave, car c'est bien d'une

${ }^{49}$ On pourrait dire que le tui exprime le fait que le pậā est, ou se veut, d'un statut supérieur au Santal à qui il s'adresse. C'est un fait que l'on s'adresse souvent (de manière qui n'est pas sans condescendance) aux Santals de cette manière. On retrouve le tui en ce sens dans la phrase 11.

50 Cette eau est de l'eau ordinaire dans laquelle on a délayé à froid de la poudre de curcuma. Le curcuma longa est l'épice la plus utilisée de la vie quotidienne, aussi bien par les Bengalis que par les Santals, dans la cuisine comme dans le rite. 
possibilité de voir son père revenir sous la forme d'un revenant qu'il s'agit alors).

Remarquons que jusqu'ici, le texte du patuã forme comme un espace sonore impénétrable. Il est seul à parler; son discours n'admet nulle pause ou interruption. Le fait d'apporter l'eau de curcuma - c'est le premier acte de la scene - vient interrompre ce flot. "L'eau de curcuma fut apportée" (haldi jal niye hala) dit le patuã. C'est un geste décisif. A partir de là, une brèche est ouverte dans les défenses du fils. De plus en plus vite, de plus en plus de choses seront demandées, et tout se passe comme s'il était impossible de ne pas les apporter. De même, à partir de là, la relation entre la famille et le patuā ne se pose plus en termes de croyance. L'emploi du prétérit (niye hala) vient marquer ce point de non-retour. Il ne s'agit plus de croire ou de ne pas croire si ce que peut dire, faire, ou être le patuā a un effet sur le défunt. Quelque chose va se passer qui dépasse ce niveau de croyance.

Le patuã reprend:

7. "Mets la dans une petite coupe. Non, ça ne va pas. Là-bas se trouvent les grandes assiettes en kansā (alliage métallique), apporte plutôt une de celles-là (tui ektā chota bāti kare rākh. Nā ei habeni. Okhāne bara kānsār thălā āche, sei ektā kare niye āy).

On retrouve ici deux énonciateurs possibles: le père et le patuā. On aurait d'abord tendance à penser au patuā. Remarquons pourtant que l'on est toujours à la deuxième personne familière (de l'impératif): le $\underline{\underline{t u i}}$ et que la troisieme phrase ("là bas se trouvent les assiettes...) fait fortement penser au père. En effet seul quelqu'un de très proche, et investi d'une certaine autorité dans la maison, oserait se référer à l'emplacement de choses aussi intérieures et particulières que sont les ustensiles en "kansa". Les assiettes faites de cet alliage métallique particulier sont celles où mangent les membres de la famille; elles sont généralement entreposées dans la cuisine, où nulle personne extérieure à la maison ne doit pénétrer; elles font normalement partie de cet ensemble d'objets qui est transmis en lignée féminine au moment du mariage. Cette phrase a par ailleurs quelque chose de familier dans le ton, si bien que l'impératif qui y est utilisé prend moins valeur d'ordre que de service (les personnes plus âgées, et plus particulièrement les 
parents envers les enfants, ont coutume de demander aux plus jeunes de faire les choses pour eux; les cadets comme les femmes y obtempèrent volontiers; l'obligation de répondre à la demande ainsi posée entre cependant toujours dans le cadre d'une relation entre deux vivants: aîné/cadet).

8. "Ton pere m'a dit: apporte une de ces assiettes en métal. Alors, le "faire voir" aura lieu. (tomār bābä boleche: sei thāla ektā kare niye eso. Sei kare dekhāno habe)

Ici, pour la première fois, le patuã se donne sans ambiguïté comme énonciateur de la phrase. Il ne parle plus au nom du père mais en son nom propre ("ton père m'a dit"). On doit remarquer qu'alors, non seulement il abandonne le tui et utilise le tomar, terme d'adresse qui convient à la personne extérieure qu'il est ${ }^{51}$, mais encore il explicite les rapports qu'il entretient entre le père et le fils: il se pose comme interlocuteur du père, émissaire de ce dernier ("ton père m'a dit"), "inter-locuteur" entre le père et le fils. Remarquons également que, alors qu'il s'exprime pour la première fois clairement en son nom, il ne dit plus: "je ferai voir", mais "le faire voir aura lieu". Tournure impersonnelle qui le met hors jeu en quelque sorte; c'est comme s'il s'éclipsait soudain pour laisser le père et le fils en présence l'un de l'autre. Notons qu'il y a encore deux façons d'interpréter ce dernier énoncé ("le faire voir aura lieu"), qui tient au fait que le verbe voir est donné a l'impersonnel. Il peut vouloir dire: 1) A travers moi, le patuã, toi [le fils] tu verras [l'image de ton père], ce qui équivaut à dire: moi, le patuă, je montrerai le pat. 2) A travers moi - le patuã -, le père verra (la vue sera donnée au père). Ce qui serait une annonce de la peinture de la pupille sur le pat. Mais, détail important, ce "voir" sans sujet reste, notons-le, conditionné au fait d'apporter des objets sur la scène: "après avoir fait ceci (c'est-à-dire avoir apporté), le faire voir aura lieu" dit le patuã. Il n'est jusqu'alors pas question de "donner". Il s'agit seulement d'apporter.

51 Cet emploi pourrait également marquer une certaine solennité dans le message. 
9. "Et ma poule jaune, elle est là ? (āmār holde murgi âche ?)

- Elle est là"

A cette soudaine question - la première depuis le début du texte que peut répondre le fils! Car on attend une réponse; le fils doit maintenant se déclarer (ce sera ses premiers mots depuis que le patuã est la). Et que peut-il dire ? Il dit, bien sur, que oui, que la poule est là. Car pourrait-il répondre autre chose à son père (que l'on croit entendre à nouveau)! Comment pourrait-il lui cacher que la poule qui lui appartient, et qu'il connaît si bien, est là ? En même temps, l'on sait clairement désormais que derrière ce "ma poule" se cache le patuã. Ainsi se trouve affirmé pour la première fois son droit - sans appel sur les objets qui sont cités.

10. "Ta belle poule jaune, il m'a dit qu'il fallait la donner. Ton pere $m$ 'a dit: donne une assiette, donne une poule, donne bien (tor bàla holde murgi dite boleche. tomär bāba boleche: ektā thāla, ektā murgi bhala kare de)

Retour au patuā comme énonciateur incontestable de la phrase, émissaire du père et interlocuteur entre le père et le fils. Deux faits sont à noter ici: 1) le patuã ne dit plus "ma poule", ni "les assiettes", mais "ta poule". Le fils est pour la première fois désigné comme propriétaire et héritier. 2) Le patuã ne dit plus "apporte", mais "donne". Cette fois, ce n'est plus une demande, c'est un ordre, et cet ordre est devenu incontournable. Ce n'est plus le service que l'on requiert familièrement d'un fills vivant, c'est quelque chose qui est de l'ordre de la contrainte: le fils doit donner ce que ce son père réclame par l'intermédiaire du patuā (sous peine de le voir revenir comme revenant).

Arrive alors le deuxième acte, sorte de "transformation" de l'acte antérieur, que d'ailleurs le patuã rappelle dans sa description: "l'assiette et l'eau de curcuma furent apportées" (sei kānsār thălă ār ektu halud jal niye hala). 
Le fait d'apporter cette assiette crée un tournant ${ }^{52}$. Aussitôt après en effet le jādu patuā prononce ses dernières paroles, en exécutant ce geste de la peinture de l'ail qui a tant retenu l'attention des commentateurs. Il "montre (ou fait ?) un peu de ? (mot quasi incompréhensibie; nous entendons quelque chose comme "mecameci)... lene ektu michāmichi( ?) dekhiye diye balla: ... $J^{53}$ nous dit notre narrateur. Cette phrase est importante. Elle indique que c'est à ce momentlà que le jādu patuã exhibe l'image et y inscrit le petit point noir de l'œil. Le mot de type onomatopéique qui est prononcé de manière presque incompréhensible (et c'est sûrement volontaire) désigne très vraisemblablement cet acte. Ce mot, nous l'avons plusieurs fois entendu employer dans les campagnes en lieu et place d'un autre terme qui est tuk-tak, et que l'on traduit habituellement par "magie". Nous sommes loin de la formulation précise et sophistiquée de caksudan.

C'est alors que le jādu patuā dit: "Regarde ton père et donne 5 roupies; donne 5 mesures de riz, donne une assiette, et les péchés qu'a commis ton père en seront réduits. Le vieux m'a dit qu'il avait fait de la pomme de terre cette année; il y en a plusieurs kilos cette année. Après avoir cultivé et engrangé, ne doit-il pas manger? Il a peiné à cultiver [et) il est mort" (dekh tor bābā ār pānctã tākā de, pānc ser cāl de ār ektā thālā de, är tor bāper yā pāp chila sab kame yābe... Bura cās kare rekhece, kābeni ? kosto kare cās karla, mare giyece)

52 Les deux objets, l'assiette et la poule, qui interviennent dans le discours du patuā et qui, il faut le noter, sont toujours représentés sur le pat, sont deux éléments centraux des rites funéraires santals. La poule fait d'abord partie des choses rassemblées près du cadavre avant sa crémation. Avant que ne soit allumé le bûcher funéraire, elle est empalée sur l'une des branches qui le forment et, comme on recouvre le cadavre du défunt de quelques bûches, le sien est recouvert de bûchettes. La poule est par ailleurs l'objet de traitements particuliers dans chacun des rites funéraires ultérieurs. L'assiette est un autre objet central du rite. Sur ce point, nous noterons seulement que, pour qu'il y ait levée définitive du deuil, il faut que le défunt accepte lors du dernier rite du deuil de prendre un dernier repas dans un tel plat (Archer, 1974: 327-342).

53 La traduction littérale de ce dernier membre de phrase est: "[ayant] apporté, il dit en montrant (ou "en donnant-montrant) un peu de...." 
Le dernier pas est fait, le patuã est arrivé à ses fins. Il dit: "regarde... et donne $(\operatorname{dekh...} \bar{a} r \ldots d e)$ "; et il n'y a pas d'échappatoire possible à ces deux ordres soudain liés. Le fils va donc regarder, et ce regard porte son consentement. Au moment où va se poser sur l'image ce regard consentant, le patuã fait ce constat, qu'il n'a jamais clairement fait auparavant: "il est mort".

Le discours du patuã, tout maladroit qu'il paraisse (il est par endroit presque inarticule) se développe donc suivant une logique élaborée. Le but est d'interpeller le fils sur la question du deuil de son père, tout en lui offrant les moyens de s'en dégager. Il était important de noter que c'est seulement après ce discours et après que des objets ont été apportés que le patuã montre le pat et effectue le "don de l'ceil". Seulement à ce moment en effet, ces objets sont ceux du mort/ceux du patuã (ou de la mort ?), cette image celle du défunt. Seulement à ce moment, le patuā peut accomplir la mission qu'il réclame pour sienne: poser dans l'œil de ce qui est dorénavant bien le défunt (et non plus seulement une image) le point noir de la pupille et emporter les objets, devenus ceux du mort/ceux du patuā.

Une grande part de l'art du patuā consiste dans son habileté à jouer sur les "personnes" du discours, qu'il prend soin d'énoncer dès le début, donnant à chacune une identité précise: le "tu" est spécifié comme étant le fils, le "je" le père, et le "il" le patuã. La donne ainsi faite, il va s'appliquer à mélanger les cartes. Très vite, l'on va se perdre entre le passé et le présent, l'ici et le là-bas, les présents et les absents, et à la fin du jeu, la donne est complètement modifiée. Le père apparaît comme un "il", et le patuā comme un "je", avant de se fondre dans l'impersonnel. Si l'on se rapporte aux travaux d'E. Benveniste (1966: 225-236), une dimension particulière apparaît alors: on voit que le père change completement de statut. Il passe de "personne" à "non-personne". Ce passage de "personne à part entière" à "non personne" ne peut cependant s'effectuer directement. Seul le passage par un je-patuā transforme le je-père en un "il", et ceci seulement dans la mesure où il y a un "tu" qui écoute. Le patuā suit le chemin inverse. Il apparaît d'abord comme un "il" (à la phrase 3) puis passe à un "je" (phrase 5), pour enfin disparaître (c'est à l'impersonnel 
qu'est mentionné son "donner à voir"). Seul dans cette histoire le statut du fils (le seul vivant concerné) ne varie pas. Du "tu" qu'il était au début du discours, "tu" il reste jusqu'à la fin. On voit aussi que dans le moment où son père apparaît comme revenant, le fils est nommément désigné comme "héritier".

Dans tout ce discours, où, au fond, trois personnages n'en font jamais que deux, l'ambiguïté est soigneusement cultivée, mais il n'est laissé aucune place à la confusion. Le patuã du moins ne la laisse jamais s'installer. A aucun moment, le fils (ou la famille du défunt) ne plonge dans l'irréel ou la folie. Il n'y a pas non plus phénomène de possession ou d'identification. La famille n'est jamais dupe de ce qui se passe. Mais le patuã règne en maître sur la situation. Cette maîtrise, il la doit, nous semble-t-il, à son habileté à faire jouer les mécanismes du deuil. Avant même de présenter le pat, il a bien en effet mis en place les éléments d'une logique de deuil. C'est sous la contrainte de cette logique que le fils s'exécute.

Il reste qu'il est nécessaire que des objets soient manipulés. On pourrait penser qu'à la limite le fils pourrait passer outre à un simple discours sur son père revenant, mais dès lors qu'il a tenu dans ses mains les objets ${ }^{54}$ que les paroles du patua $\bar{a}$ vont transformer en choses funéraires, il s'avère impossible de résoudre la situation autrement que par leur abandon. Ces objets sont devenus des dûs. C'est bien quelque chose du deuil qui est mis en place par le patuā. Quant au fils, il ne peut s'en sortir, sauf à passer par le dictat patuā.

54 On ne peut s'empêcher de penser ici à la façon dont procèdent certains camelots. Pour peu que l'on ait été "harponné" par le discours que toujours ces derniers déploient, on sait combien on peut se retrouver les bras chargés d'objets dont on n'avait nullement souhaité la présence. Il faut alors parfois faire un réel effort sur soi pour s'en débarrasser. Tout au moins en garde-t-on souvent au moins un! Le jadu patuã utilise quelque chose de cette technique. Notre narrateur remarqua ainsi (cf. plus bas) que si la famille du défunt ne donne pas toujours tout ce que le patuã réclame, elle donne toujours "au moins quelque chose". N'oublions pas enfin qu'un certain nombre de Bengalis disent que le jādu patuā est un charlatan. Il est toutefois évident que quelque chose de particulier se joue dans son cas. Il ne s'agit pas en effet de n'importe quels objets, et le but du pạ uā est non de les faire désirer mais au contraire de les rendre indésirables. 
Nous sommes loin, nous semble-t-il, de la proposition simple selon laquelle le comportement de la famille du défunt serait uniquement motivé par de la crainte. Nous ajouterons qu'il n'est pas impossible qu'à la limite, le jādu pațā puisse se passer de la présentation de l'image du défunt pour que s'accomplisse, aux yeux des Santals, son travail funèbre. C'est peut-être ce qu'indique la remarque d'un jádu patuâ, rapportée par J. B. Faivre, selon laquelle "il recevrait comme d'habitude" s'il faisait sa tournée sans ses rouleaux (Faivre, 1980: 113). C'est aussi ce que peut vouloir dire le commentaire de notre narrateur patuā à propos cle la dernière phrase du discours tenu par son compagnon "magicien". Après: "Regarde ton père et donne 5 roupies; donne 5 mesures de riz... [Le vieux] a peiné à cultiver [et maintenant] il est mort" le jādu patuā avait ajouté: "Moi, j’ai un maître (guru). Pour ton pere, j'effectuerai le travail"55. Et notre patuã de commenter aussitôt: "Les Santals ne comprennent pas ces paroles. Ils n'ont pas de guru. Tout ce qu'ils comprennent, c'est qu'une assiette, un pot, sont demandés, et cela sera donné. Ils ne donneront pas tout ce qui est demandé, mais ils donneront toujours quelque chose"56. Ce qui semble important aux yeux des Santals est donc le fait que ce qui est réclamé soit donné ${ }^{57}$. Là réside la contrainte essentielle entre le père et le fils tant que le premier n'est pas "entré dans le royaume de Yama". C'est toute l'irritante habileté du patuā que d'oser jouer de cette situation en dehors du rite qui régle mente cette contrainte. Qui peut jouer du rite en dehors du rite!

Il faut avant de terminer faire un dernier commentaire. Nous avons dit que le patuā se rend chez les Santals. On peut se demander pourquoi il ne visite pas les foyers hindous (ou musulmans). Nous

55 Amār guru âche. Tomär bābär janye käj kare debo.

56 Là encore, la forme du verbe utilisé par le patuā ne permet pas d'établir avec certitude qui demande. La phrase bengalie est: thálä, bāti, ya (ou ye ?) cãibe, sei debe..., mot à mot: "une assiette, un pot, ce qui sera voulu (ou ce qu'il désire), cela il donnera". Qui est ce "il" qui "veut" ? Le palıã ? Le défunt ? Yama ?

${ }^{57}$ La présentation de l'image et le placement de l'oeil dans la pupille étant comme un acte de ratification (de l'état de fait établi par le discours patuã). 
ferons à ce sujet une seule remarque: chez les Santals, les morts parlent. Ils s'adressent aux vivants. Les spécialistes de cette société nous apprennent notamment qu'à différents moments des rites funéraires, le défunt s'exprime à travers l'un des deuilleurs au cours de mises en scène rituelles ${ }^{58}$. "L'acteur qui joue le mort demande de l'eau... On le questionne sur la façon dont il est mort. Il répond à la première personne, par exemple: "Je suis parti de mon propre chef..." (Archer, 1974: 334). Jusqu'au dernier rite du cycle funéraire, il y a de telles mises en scène. A ce moment, le deuilleur principal joue le rôle du défunt, tandis que deux autres jouent des personnages divins. Il demande de l'eau, et finalement on le nourrit pour la dernière fois. Le défunt est ainsi "ré-identifié" dit Archer (idem, 336) ${ }^{59}$. Il semble par ailleurs admis que pendant tout un temps (nous allons voir lequel), le défunt (dont les cendres ont été déposées aux limites du village dans un pot dont l'orifice n'a pas été completement bouché afin qu'il puisse "aller et venir" librement) puisse revenir rôder parmi les siens, s'adresser à sa famille ou aux membres de son village, se faire voir d'eux, leur parler. C'est une chose redoutée mais non maligne. La période la plus sensible de ce point de vue semble être celle qui s'étend entre la crémation du corps (qui a lieu le jour du déces) et le rite dit "du retour des cendres" dans la maison du défunt. Ce rite a lieu l'après-midi du 6ème jour (après le décès); il est obligatoirement précédé du rite tel nāhan (onction avec l'huile); au terme du tel nāhan en effet, le défunt "a perdu l'ennemi qui l'a tué"60. Ce serait pendant cette période que, si l'on lit entre les lignes du texte de W. G. Archer (1974: 335), le patuã se présenterait devant la maison en deuil ${ }^{61}$.

58 Pour le détail des rites funéraires santals, nous avons consulté Archer, 1974: 327-342, Boucz, 1985: 127-130 et Carrin-Bouez, 1977.

$59 \mathrm{~S}$. Bouez (1985: 128) parle quant à lui de "possession".

${ }^{60}$ Les cinq premiers jours après le décès, le défunt est supposé demeurer, aux limites du village, auprès du "meurtrier qui l'a tué".

61 A l'issue du rite de "retour des cendres", le défunt n'est toutefois "pas encore admis dans le royaume des morts". Il demeure dans la maison en deuil, sous la forme d'une poignée de cendres placées dans un pot neuf, ce que les Santals désignent comme étant les "fleurs de l'os". Il reste là, "en transit", pendant quelques semaines. Le deuil est levé pour le village, mais non pour les parents. Ceux-ci doivent attendre 
Rien de semblable ne se passe chez les Hindous. Le cadavre est traité avec la plus grande rapidité, la crémation se fait sans tarder, tout le rite semble s'ingénier à "verrouiller" ce qui pourrait donner lieu à un trop grand rapprochement avec le défunt. Toute manifestation de ce dernier est considérée comme anormale. Entendre parler le mort, faire parler le mort ou encore parler pour lui serait déjà avoir affaire à un bhüt, revenant ou un esprit malfaisant.

Nous finirons en nous interrogeant sur ce que représente le patuă. Nous avons vu qu'il se situe comme un intermédiaire, inter/locuteur entre le père et le fils. On dit aussi que les objets qu'il reçoit - assiette (pour la nourriture), pot (pour l'eau), ombrelle (pour se protéger du soleil)... -, sont destinés à être remis par son intermédiaire au père défunt pour le voyage que ce dernier est censé effectuer vers le royaume des morts. En effet, ces objets constituent bien le nécessaire à un voyage. W. G. Archer remarque par ailleurs que le jădu patuă "joue le rôle d'un mendiant funéraire (funerary beggar)" (op. cité p. 335). Il fauî toutefois remarquer que ce statut n'implique pas celui de "magicien", qui caractérise le jādu patuã. On peut se demander si le jădu patuã ne serait pas perçu comme Yama lui-même, dieu très présent, nous l'avons vu, dans l'eschatologie santale. La relecture de L. Renou inviterait à cette conclusion. Nous avons montré que le rôle funèbre du jādu patuã réside dans son habileté à contraindre. Or, nous dit Renou, "les textes interprètent le nom de Yama par le verbe yam, "contraindre" (1947, I: 495). Ce funeste personnage qu'est le patuā ne participe-t-il pas de la nature même de Yama, de ce Yama qui, rappelle encore Renou, a précisément, lui aussi, "de grands pouvoirs magiques". Enfin, tout comme le jădu patuã se place en dehors de la

deux autres cérémonies: 1) celle où, les "fleurs de l'os" ayant été déposées dans une rivière, le défunt devient bonga (ancêtre, esprit); 2) le rite dit bhandan (terme que nous traduisons par "attaché, lié"), séparé du précédent par neuf jours minimum, au cours duquel le mort s'exprimera pour la dernière fois dans une mise en scène rituelle par la bouche de l'un des deuilleurs. Il serait intéressant de préciser à quel moment le patıã cesse de pouvoir intervenir. Sa venue est-elle liéc à l'idée que le défunt réside pendant quelques jours aux limites du village auprès du "meurtrier qui l'a tué" ? Les informations dont nous disposons ne sont pas suffisantes pour éclaircir ces points. 
société (il est rejeté des Santals mais il est aussi rejeté par les Hindous), sa pratique se place en dehors des systèmes rituels auxquels elle s'adresse ou dont elle emprunte les outils; et il y a de quoi hésiter sur le statut à accorder à ses paroles. N'est-ce pas aussi cela qui le place dans l'ordre du jädu. Il reste que le jādu patuā a su articuler autour d'un axe commun (la contrainte) des représentations contrastées de la mort: celles, hindoues, où la mort se reconnait dans une écriture, et celles, santales, où elle se fait entendre directement par la voix des morts eux-mêmes.

R. de Selva

E.P.H.E. 


\section{Références bibliographiques}

\section{Archer, Mildred}

1977 Indian Popular Paintings in the India Office Library, London, 196 p., 92 pl.

Archer, W. G.

1974 The Hill of Flutes. Life, Love and Poetry in Tribal India. A portrait of the Santals. Londres, G. Allen \& Unwin.

Articles pat, patuā et patkar dans:

Haughton, Sir Graves

1833 A dictionary of bengali and sanskrit, London.

Mitra, Subal Chandra

1971 Saral bānglā abhidān, Calcutta (1906).

Bāna

Harsacarita, texte sanscrit traduit en anglais par Cowell, 1897.

Benveniste, Émile

1966 Problèmes de linguistique générale, Paris, Gallimard, 345 p.

Bouez, Serge

1985 Réciprocité et hiérarchie. L'alliance chez les Ho et les Santals de l'Inde, Paris, Société d'ethnograhie.

Carrin-Bouez, Marine

1977 "Les pierres de mémoure. Rites funéraires des Ho du Bihar et de l'Orissa", Objets et mondes, XVII, 3.

Chatterjee, Suniti Kumar

1975 The Origin and Developpement of Bengali Language, Rupa \& $\mathrm{C}^{\circ}$, Calcutta, 3 vol.

Dutt, Gurusaday

1932 "The Art of Bengal", The Modern Review, mai 1932, pp. 519529.

1932 "The Tigers God in Bengal Art", The Modern Review, nov. 1932, pp. 520-529.

1939 Patuā sāngit ("Chants patua"), Calcutta, Calcutta University Press.

Faivre, Jean-Baptiste \& Chakraborty, Utpal

1980 "Rouleaux peints des chitrakars ou jadu patuas des Santal Parganas. Etat du Bihar (Inde)", Objets et Mondes, 20, 3. 
Malamoud, Charles

1989 Conférence 1988-1989, Annuaire de l'Ecole Pratique des Hautes Etudes, tome XCVII, 125-129.

1991 Exposé: "Les aspects rituels de la relation frère-sœur dans l'Inde ancienne", Collège de France, 16/01/1991.

Monnier-Williams Sir

1899 Sanskrit English Dictionnary, New ed., Oxford Clarendon Press/Oxford, Univ. Press (Réimpr. 1964).

Ostör, Akos

1980 The Play of the Gods, University of Chicago Press, $241 \mathrm{p}$.

Renou Louis \& Filliozat, Jean

1947 L'Inde classique, tome I, Payot, Paris.

Singha, Siri Guna

1957 La rechnique de la peinture indienne d'après les textes du Silpa, PUF (Annales du Musée Guimet) t. LXIII.

Talwar, Kay \& Krishna, Kalyan

1979 Indian Pigments Paintings on Cloth, Ahmedabad (Historic textiles of India at the Calico Museum, v.III).

Toffin, Gérard

1990 "Hiérarchie et idéologie du don dans le monde indien", L'Homme, 114, XXX, 2, 130-142.

Wilkins, W.

1975 Hindu Mythology, Rupa \& $\mathrm{C}^{\circ}$, Calcutta (lère éd. 1882), 499 p. 